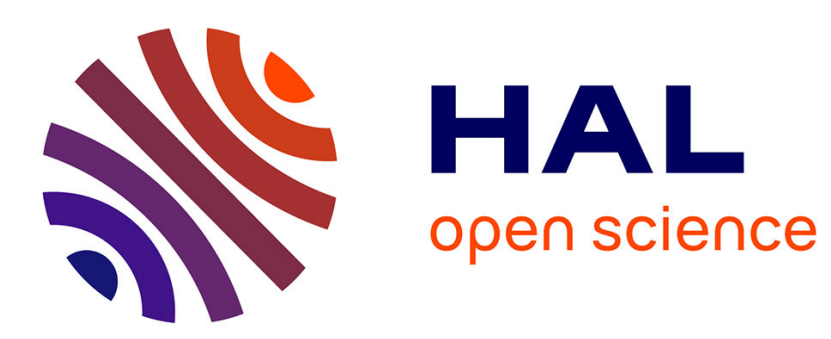

\title{
Modeling the annual cycle of HDO in the Martian atmosphere
}

\author{
Franck Montmessin, T. Fouchet, François Forget
}

\section{To cite this version:}

Franck Montmessin, T. Fouchet, François Forget. Modeling the annual cycle of HDO in the Martian atmosphere. Journal of Geophysical Research. Planets, 2005, 110 (E3), pp.E03006. 10.1029/2004JE002357 . hal-00078180

\section{HAL Id: hal-00078180 https://hal.science/hal-00078180}

Submitted on 30 Jan 2016

HAL is a multi-disciplinary open access archive for the deposit and dissemination of scientific research documents, whether they are published or not. The documents may come from teaching and research institutions in France or abroad, or from public or private research centers.
L'archive ouverte pluridisciplinaire HAL, est destinée au dépôt et à la diffusion de documents scientifiques de niveau recherche, publiés ou non, émanant des établissements d'enseignement et de recherche français ou étrangers, des laboratoires publics ou privés. 


\title{
Modeling the annual cycle of HDO in the Martian atmosphere
}

\author{
F. Montmessin ${ }^{1}$ \\ NASA Ames Research Center, Moffett Field, California, USA
}

T. Fouchet

LESIA, Observatoire de Paris, Paris, France

F. Forget

Laboratoire de Météorologie Dynamique, Institut Pierre Simon Laplace, Paris, France

Received 8 September 2004; revised 30 November 2004; accepted 22 December 2004; published 24 March 2005.

[1] We present the results of the first three-dimensional (3-D) simulation of the water isotope HDO in the Martian atmosphere. This deuterated isotope of water has long been used on both Earth and Mars as a proxy to understand the climatic evolutions of these planets. On Mars, the current enrichment in deuterium concentration in the atmosphere is believed to be indirect evidence of a wetter climate in the past. Due to its vapor pressure being lower than that of $\mathrm{H}_{2} \mathrm{O}, \mathrm{HDO}$ gets fractionated at condensation and therefore concentrates in the Martian water ice clouds. Our study aims at understanding the latitudinal, vertical, and temporal variations of this species under current Martian climate. Our results indicate that the globally averaged $\mathrm{D} / \mathrm{H}$ ratio in the Martian atmosphere should vary modestly with season, with changes on the order of $2 \%$. Locally, however, this same ratio exhibits large annual changes (by a factor of 2) in the high-latitude regions. These fluctuations are controlled by the Polar Hood water ice clouds, within which HDO gets heavily fractionated. Due to the combined action of summer clouds above the north polar cap and to the cold-trapping effect of the south residual cap, the global atmospheric deuterium concentration is predicted to be more than $15 \%$ lower than the concentration in the north permanent cap ice. We thus extrapolate by suggesting that the "true" D/H ratio of Martian water may exceed 6.5 (wrt. SMOW), rather than the 5.6 inferred from atmospheric probing. The globally and annually averaged vertical distribution of HDO exhibits a mild decline with altitude, a result in significant contrast with previous 1-D studies. These results will help constrain more accurately the photochemical models aimed at understanding the observed low concentration of deuterium at high altitudes and thus the process of water escape to space.

Citation: Montmessin, F., T. Fouchet, and F. Forget (2005), Modeling the annual cycle of HDO in the Martian atmosphere, J. Geophys. Res., 110, E03006, doi:10.1029/2004JE002357.

\section{Introduction}

[2] The presence of channels, valley networks, and ancient lakes at the surface of Mars [Baker et al., 1992; Mangold et al., 2004] provides evidence for a hydrological cycle early in the Martian history, when the planet possibly harbored a warmer and wetter climate than the present conditions. The amount of water that once flowed on Mars remains controversial but most estimates agree on a 500-m layer averaged over the entire planet [Baker et al., 1992]. Since then, water has been segregated between various known reservoirs (the atmosphere, the seasonal polar caps, the permanent polar caps, and the water ice in the shallow

\footnotetext{
${ }^{1}$ Now at Service d'Aèronomie, CNRS/IPSL, Paris, France.
}

Copyright 2005 by the American Geophysical Union. 0148-0227/05/2004JE002357 subsurface of the high-latitude regions), and other putative reservoirs such as deep crustal water. Some water was also definitively lost to space.

[3] One clue to constrain the relative sizes of these different water reservoirs is the water $\mathrm{D} / \mathrm{H}$ ratio in the current Martian atmosphere [Owen et al., 1988; Yung et al., 1988; Kass and Yung, 1999; Krasnopolsky and Feldman, 2001]. The $\mathrm{D} / \mathrm{H}$ ratio in atmospheric water is enriched by a factor of 5.6 compared to the terrestrial ratio [Owen et al., 1988; Krasnopolsky et al., 1997]. This deuterium enrichment is thought to result from the preferential escape of hydrogen atoms over deuterium atoms, characterized by a fractionation factor $F$ in the hydrogen escape. The $\mathrm{D} / \mathrm{H}$ is hence a measure of the ratio of the current exchangeable water reservoir to the initial exchangeable water reservoir. From the analyses of SNC meteorites (shergottite-nakhlite-chassignite), it seems even possible to 
record the evolution with time of the Martian water reservoirs. Using an ion microprobe, Watson et al. [1994] and Leshin [2000] showed that these Martian meteorites are also enriched in $\mathrm{D}$, with a factor ranging from 2 times to 5.5 times the terrestrial ocean concentration. Watson et al. [1994] interpreted these high $\mathrm{D} / \mathrm{H}$ ratios as an early postcrystallization $\mathrm{D}$ enrichment by crustal fluids with atmospheric $\mathrm{D} / \mathrm{H}$ ratios. This implies that near the crystallization time of SNCs, up to $1.3 \mathrm{Gyr}$ ago, the atmosphere bore a $\mathrm{D} / \mathrm{H}$ ratio similar to the present value.

[4] It is therefore important for our understanding of the history of Martian water to accurately estimate the isotopic fractionation factor $F$ describing the relative escape of deuterium onto that of hydrogen. However, the D atoms that populate the upper atmosphere where they can escape to space, are the end-products of a complex photochemical cycle within which the HDO molecules of the lower atmosphere are the sole precursors [Yung et al., 1988]. A compilation of previous studies dedicated to Martian HDO shows that the differential escape of $\mathrm{H}$ and $\mathrm{D}$ results from three different processes: (1) a mass difference that favors upward molecular diffusion and thermal escape of hydrogen [Yung et al., 1988], (2) the preferential photolysis of $\mathrm{H}_{2} \mathrm{O}$ over HDO [Cheng et al., 1999], (3) the Vapor Pressure Isotope Effect (VPIE) that produces an isotopic fractionation at condensation [Krasnopolsky, 2000; Bertaux and Montmessin, 2001]. First studied by Fouchet and Lellouch [2000], this latter process results from the slight difference between the vapor pressures of $\mathrm{H}_{2} \mathrm{O}$ and HDO. During water condensation, the solid phase is enriched in deuterium to the expense of the vapor phase. This effect can be significant and may reduce the $\mathrm{D} / \mathrm{H}$ ratio above the condensation level to values as low as $10 \%$ of the $\mathrm{D} / \mathrm{H}$ ratio near the surface [Fouchet and Lellouch, 2000; Bertaux and Montmessin, 2001].

[5] When the hygropause is located below the HDO photolysis peak, VPIE couples with the fractionation of HDO at photolysis. In this case, the water vapor that is photodissociated has already been depleted in deuterium during its ascent through the hygropause. As photolysis itself discriminates HDO so that less deuterium-bearing molecules (mostly HD) are produced, this combination of processes should dramatically restrain the production of $\mathrm{D}$ atoms, eventually reducing their escape rate to space relatively to $\mathrm{H}$ atoms. This coupling has been advocated by Bertaux and Montmessin [2001] to explain the measurements of Krasnopolsky et al. [1998] which showed an unexpected paucity of D atoms at high altitude $(>100 \mathrm{~km})$.

[6] The above estimated D depletion was based on 1-D modeling using mean meteorological conditions. However, the Martian meteorological fields (atmospheric temperature, water column density) vary strongly with season, local time, and location. For instance, the altitude of the hygropause is known to vary from $10 \mathrm{~km}$ up to $\sim 60 \mathrm{~km}$ [Smith, 2002] (and could even be closer to the surface in the winter polar regions). Planetary-scale atmospheric motions, like traveling waves or the overturning circulation, should also affect the local deuterium content of atmospheric and precipitating water. In short, HDO must have its own cycle as it is the case on Earth [Joussaume et al., 1984], though closely related to the $\mathrm{H}_{2} \mathrm{O}$ cycle but with some differences due to the condensation-induced fractionation. Within this context, the HDO cycle can only be addressed consistently by comprehensive three-dimensional models, whereas the simple models of Fouchet and Lellouch [2000] and Bertaux and Montmessin [2001] are limited by their 1-D representation of atmospheric processes. As a result, the fractionation coefficient $F$ derived by Krasnopolsky [2000] and Krasnopolsky and Feldman [2001] on the basis of these earlier studies still suffers from significant uncertainties.

[7] The $\mathrm{D} / \mathrm{H}$ ratio heterogeneity over the planet induced by the VPIE may also affect the interpretation of the $\mathrm{D} / \mathrm{H}$ ratios measured in SNC meteorites. Indeed, if the atmospheric deuterium content varied from place to place, the crustal water may also reflect such variations. Different $\mathrm{D} / \mathrm{H}$ ratios measured in different meteorites could thus result from different $\mathrm{D} / \mathrm{H}$ ratios in crustal water rather than different degrees of alteration.

[8] Here, we present the first simulation of the HDO seasonal cycle using a General Circulation Model (GCM). The goals of this study are to explore the fate of HDO in the Martian atmosphere, to understand its seasonal and geographical distributions in view of a more rigorous assessment of the production of deuterium atoms that will ultimately escape to space. Two separate fractionation cases are analyzed in order to bound the global effect of VPIE on the annual cycle of HDO. Since the number of HDO measurements in the Martian atmosphere remains quite low, it is still difficult to constrain accurately the validity of our results. However, we shall see that our work provides significant new constraints on the exchanges of deuterium between the atmospheric and the surface reservoirs, increasing our comprehension of the deuterium cycle as a whole.

\section{Model Overview}

[9] This study on HDO has been performed with the Martian General Circulation Model developed at Laboratoire de Météorologie Dynamique. This MGCM is a grid-point model predicting the evolution of the usual meteorological variables in the Martian atmosphere (surface pressure, zonal and meridional winds and potential temperature). Extensive documentation of the model is given by Forget et al. [1999]. The grid discretization used in our study corresponds to a horizontal resolution of $5.6^{\circ}$ in longitude and $3.7^{\circ}$ in latitude, whereas 25 vertical layers are used to represent the atmospheric shell from the surface up to a height of around $90 \mathrm{~km}$. Radiative transfer accounts for scattering, absorption and emission by $\mathrm{CO}_{2}$ and dust particles in both visible and infrared spectral intervals. A recent development of the MGCM includes the implementation of a spatially and temporally prescribed amount of airborne dust in each model box. This "dust scenario" has been designed by adjusting the dust distribution so as to match a large set of temperature profiles inferred from Mars Global Surveyor observations (Forget et al., 2004, manuscript in preparation).

[10] Our model benefits of a representation of waterrelated processes, a version of which being described by Montmessin et al. [2004]. It includes the major processes affecting water vapor in the Martian atmosphere (except regolith adsorption); e.g., transport by winds, exchanges with the surface, atmospheric condensation and sublimation as well as sedimentation of icy particles. Water can either sublime (if ice is present on the ground) or condense onto 
the surface depending on the difference in mixing ratios between the vapor in the first layer and that in contact with the surface. Water ice clouds are supposed to form whenever water gets supersaturated with respects to ice. In that case, the predicted amount of condensate is spread uniformly over a prescribed number of dust nuclei, allowing one to deduce a mean radius for the icy particles. This radius value is then used to compute cloud particle sedimentation rates. Additionally, atmospheric tracers are vertically mixed by the GCM-predicted turbulent motions as well as in convectively unstable layers.

[11] This model shows itself successful at reproducing the observed seasonal hydrological cycle on Mars, supporting its use for the investigations of peripheral subjects of interest (like the first three-dimensional simulation of ozone on Mars by Lefèvre et al. [2004]). At some specific seasons and locations, however, the fit to data can be poor, especially in the southern hemisphere summer and spring where the simulated water vapor content does not decrease as rapidly as observed. Montmessin et al. [2004] have suggested that regolith adsorption could play a role to explain this discrepancy. They also mentioned that the modeled $\mathrm{CO}_{2}$ cap retreats too quickly in the southern hemisphere, affecting the release and transport of water during summer. These differences remain nonetheless minor in comparison of what is otherwise an overall good agreement between model and observations.

\subsection{Treatment of HDO}

[12] In the model, HDO is represented in both its vapor and icy phases, and is submitted to all the processes previously described for $\mathrm{H}_{2} \mathrm{O}$. However, during condensation only, we account for the same fractionation effect described by Fouchet and Lellouch [2000] and Bertaux and Montmessin [2001]. Condensation-induced fractionation has been experimentally measured by Merlivat and Nief [1967], but in a restricted temperature range which is significantly warmer than Martian typical conditions. The resulting fractionation factor $\alpha$, which represents the relative concentration of HDO in ice onto that in the surrounding vapor at thermodynamic equilibrium is given by Merlivat and Nief [1967] as

$$
\alpha=\frac{\left(\mathrm{HDO} / \mathrm{H}_{2} \mathrm{O}\right)_{\text {ice }}}{\left(\mathrm{HDO} / \mathrm{H}_{2} \mathrm{O}\right)_{\text {vap }}}=\exp \left(\frac{16288}{\mathrm{~T}^{2}}-9.34 \times 10^{-2}\right) .
$$

Merlivat and Nief [1967] indicate that their inferred fractionation law exhibits the same temperature dependence as that predicted by quantum mechanics. On this basis, we have assumed this expression for $\alpha$ to be valid for a broader range of conditions and thus we have extrapolated it down to Martian temperatures. Accordingly, this relation gives a relative enrichment of deuterium in ice compared to vapor of $72 \%, 51 \%$ and $37 \%$ at $160 \mathrm{~K}, 180 \mathrm{~K}$ and $200 \mathrm{~K}$ respectively.

[13] A significant issue concerns the diffusivity of HDO molecules in ice. It has been advocated by Jouzel and Merlivat [1984] that HDO diffusivity is too slow to permit isotopic homogenization within an icy crystal under terrestrial conditions. This statement implies that an isotopic equilibrium can only be achieved between vapor and the mass flux of condensation (the term "equilibrium" referring to the state where no net isotopic flux is exchanged between two phases), regardless of the isotopic content of the preexisting bulk of ice. In contrast, the diffusivity of HDO molecules in liquid water is large enough such that isotopic equilibrium can be obtained between a bulk of liquid water and its gaseous environment. Suppose, to clarify ideas, that during an episode of condensation, an amount of water $d M_{h 2 o}$ and heavy water $d M_{h d o}$ is exchanged between the vapor phase $M^{v}$ and the condensed phase $M^{c}$. If the condensed phase of water is liquid, then we have the following relation:

$$
\alpha=\frac{\left(M_{h d o}^{c}+d M_{h d o}\right) /\left(M_{h 2 o}^{c}+d M_{h 2 o}\right)}{\left(M_{h d o}^{v}-d M_{h d o}\right) /\left(M_{h 2 o}^{v}-d M_{h 2 o}\right)},
$$

so that

$$
d M_{h d o}=\alpha \frac{\left(M_{h 2 o}^{v}-d M_{h 2 o}\right)\left(M_{h 2 o}^{c}+d M_{h 2 o}\right)}{M_{h d o}^{v}-d M_{h d o}}-M_{h d o}^{c} .
$$

If the condensed phase is ice, we have this time

$$
\alpha=\frac{d M_{h d o} / d M_{h 2 o}}{M_{h d o}^{v} / M_{h 2 o}^{v}}
$$

and

$$
d M_{h d o}=\alpha \frac{M_{h 2 o}^{v} d M_{h 2 o}}{M_{h d o}^{v}} .
$$

If an air mass is progressively cooled such that a fraction $x$ of its initial water vapor content $\left(V_{h 2 o}^{O}\right)$ is brought to condensation, then in the case of vapor to liquid transformation and assuming that $\alpha$ does not change with temperature, the remaining water vapor amount $\left(V_{h 2 o}^{x}\right)$ will exhibit an isotopic ratio of

$$
\frac{V_{h d o}^{x}}{V_{h 2 o}^{x}}=\frac{1}{1+x(\alpha-1)} \frac{V_{h d o}^{o}}{V_{h 2 o}^{o}},
$$

whereas if the transformation consists of vapor turned into ice, the isotopic ratio of the remaining fraction of vapor should be

$$
\frac{V_{h d o}^{x}}{V_{h 2 o}^{x}}=(1-x)^{\alpha-1} \frac{V_{h d o}^{o}}{V_{h 2 o}^{o}} .
$$

Figure 1 shows the dependence of $\left(V_{h d o}^{x} / V_{h 2 o}^{x}\right)$ to $x$ for both expressions. As expected, the process of solid condensation produces a much higher decrease of deuterium in vapor than liquid condensation. This type of fractionation leads to the creation of an isotopic gradient in the ice particle (with higher concentration of HDO near the particle core) that HDO diffusivity is too slow to relax when condensation and sedimentation processes are relatively fast. This has already been discussed by Dansgaard [1964], who assimilated fractionation during solid condensation as a process of Rayleigh distillation. If Rayleigh distillation dominates isotopic exchanges during the formation of water ice clouds on Earth [Jouzel and Merlivat, 1984], it appears, however, not to be the case on Mars. Indeed, the measured value of HDO molecular diffusivity in ice $\left(\sim 10^{-14} \mathrm{~m}^{2} \mathrm{~s}^{-1}\right)$ yields a characteristic timescale for the migration of HDO molecules inside a micron-sized particle (typical size of Martian ice 


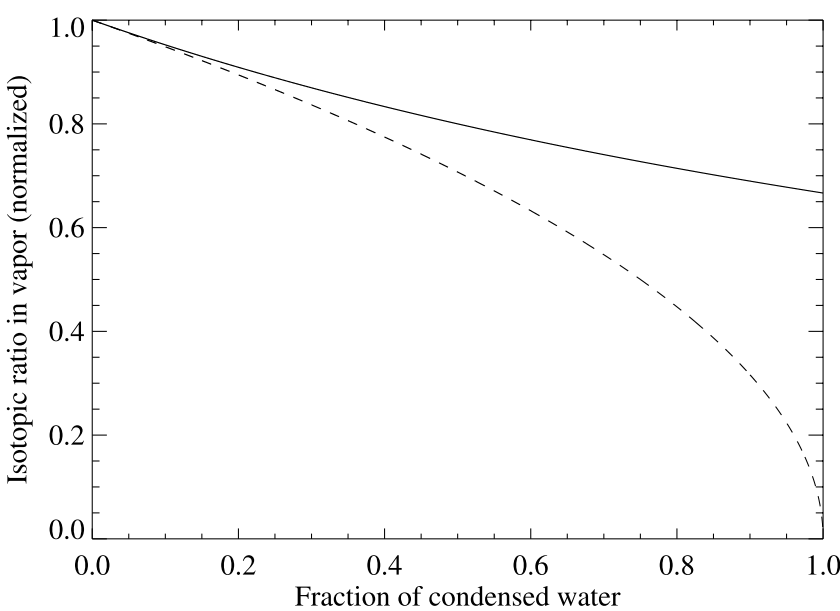

Figure 1. Evolution of the water isotopic ratio in a gaseous sample brought to condensation at a temperature of $180 \mathrm{~K}$. Two different fractionation processes are plotted here. The dashed line represents the case where only the instantaneous flux of condensation is at isotopic equilibrium with the gaseous phase. The solid line is for the case of an isotopic equilibrium between the whole condensed phase and the surrounding gas.

crystals) of the order of 100 seconds. This value intersects atmospheric condensation and sedimentation timescales on Mars, which range from seconds to days depending on altitude and crystal size [Michelangeli et al., 1993]. Representing the competition between condensation/sedimentation and HDO diffusion in ice is, however, beyond the capability of our model as it supposes to store the isotopic gradient of ice particle individually, a quantity that depends on the history of each particle.

[14] For this reason, we have followed the approach employed by Fouchet and Lellouch [2000] who separately explored two methods of fractionation in their modeling of HDO in the Martian atmosphere. We will therefore concentrate on two idealized cases: a Rayleigh Distillation case (hereafter referred to as RD) where isotopic exchange is represented by equation (2), and a Rapid isotopic Homogenization case (hereafter referred to as $\mathrm{RH}$ ) where isotopic exchange is computed following equation (1).

[15] Nevertheless, in the case of direct condensation of water onto the Martian surface, HDO fractionation is supposed to follow equation (2), regardless of the type of fractionation chosen to prevail in the atmosphere. This assumption is motivated by the fact that seasonal frost deposits are generally thick enough (on the order of $100 \mu \mathrm{m}$ ) so that HDO diffusion in the icy layer can be neglected.

\subsection{Simulation Setup}

[16] In our model, the north residual cap is represented by an infinite source of water ice at the north pole with a southern boundary located at $80^{\circ} \mathrm{N}$. At the south pole itself, temperature is set to follow carbon dioxide dew-point so as to mimic the residual $\mathrm{CO}_{2}$ cap. Simulations are started with an initially dry atmosphere, and are run until a water cycle in steady state is obtained. As done by Montmessin et al. [2004], the water cycle is considered being in steady state if the interannual changes in the global water vapor inventory are less than $1 \%$; a value obtained after 15 years of simulation. As it is for water, the north residual cap is assumed to constitute the unique source of HDO on the Martian surface. However, in the absence of measurements of the concentration of deuterium residing in the cap, we prescribed the $\left(\mathrm{HDO} / \mathrm{H}_{2} \mathrm{O}\right)$ ratio in the permanent cap at $1.7 \times 10^{-3}$; i.e., the observed global concentration of HDO in the Martian atmosphere [Krasnopolsky et al., 1997].

\section{Predicted Cycle of HDO: The Rayleigh Distillation Case}

[17] We now discuss the results obtained by the model in the specific case of fractionation where only the condensation flux can be at isotopic equilibrium with the surrounding vapor; i.e., the Rayleigh Distillation case. As explained previously, this type of fractionation should lead to the largest removal of HDO molecules in the vapor phase when clouds are forming.

\subsection{Seasonal Cycle of HDO}

[18] It can be expected that the HDO seasonal cycle qualitatively matches that of water as the two species only differ from each other by a slight shift in their respective vapor pressure curve. The HDO cycle is displayed in
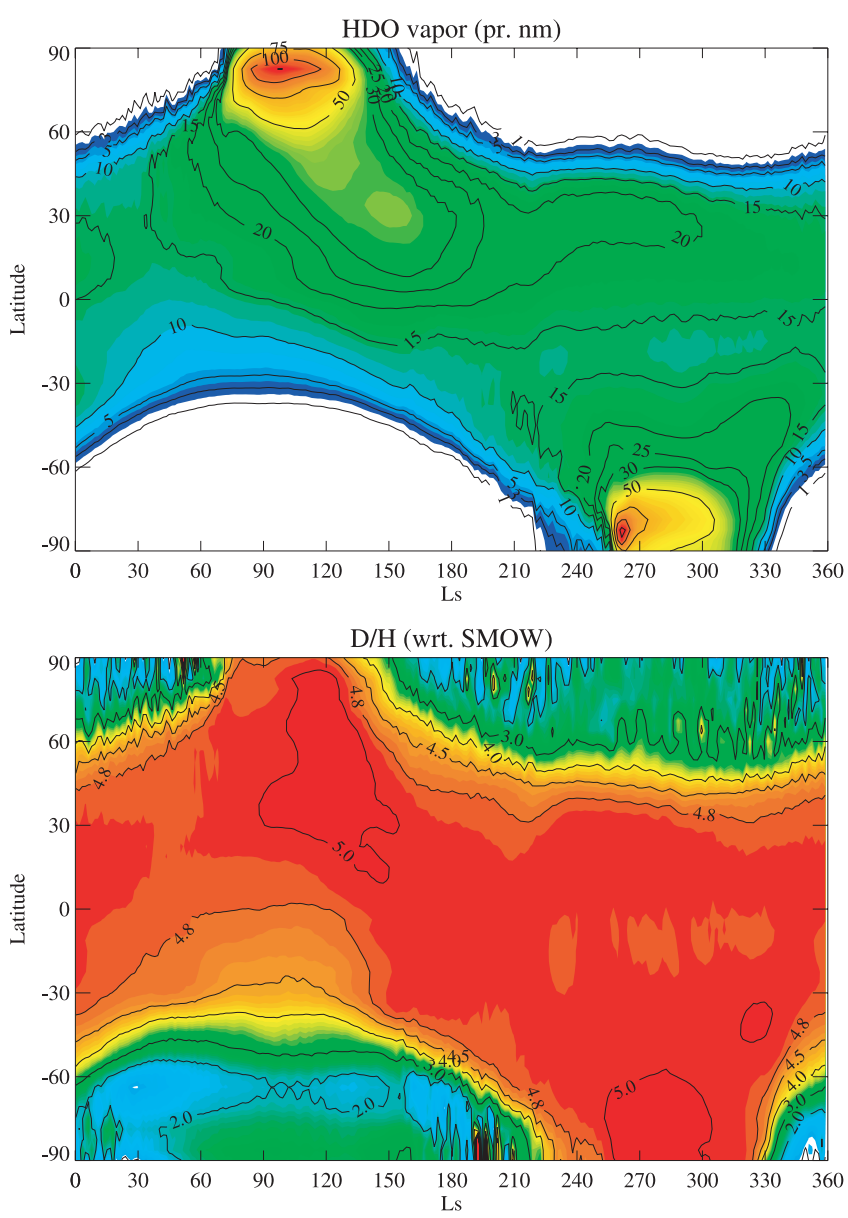

Figure 2. (top) Latitudinal and seasonal distribution of the zonally averaged abundances of HDO vapor in the Martian atmosphere as predicted by the model. (bottom) Corresponding values of the $\mathrm{D} / \mathrm{H}$ ratio in the vapor phase. 

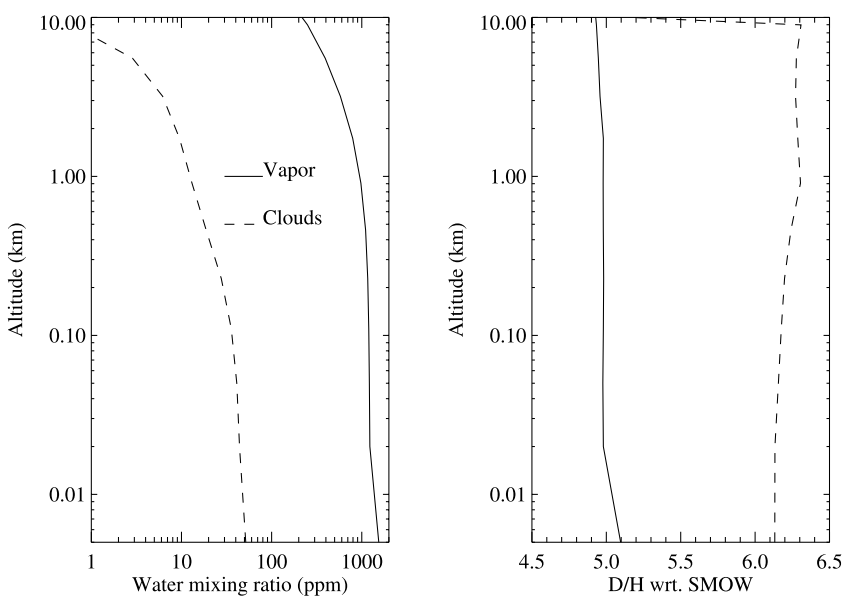

Figure 3. The left-hand plot gives the averaged vertical distributions of water vapor and clouds in the region poleward of $80^{\circ} \mathrm{N}$ at $\mathrm{L}_{s}=90^{\circ}$. The right-hand plot gives the corresponding $\mathrm{D} / \mathrm{H}$ values in each phase. It can be seen that the presence of atmospheric water ice at low heights yields a decrease of the $\mathrm{D} / \mathrm{H}$ ratio in water vapor.

Figure 2, where the column-integrated abundances of HDO are reported as a function of time and latitude (in precipitable nanometers, henceforth pr. nm). Our predicted HDO cycle exhibits the same typical trends as those already observed for the water cycle [Jakosky and Farmer, 1982; Smith, 2002, 2004]. As an example, we note that HDO abundances peak above the north permanent cap in spring and summer, when large amounts of both water and heavy water are released into the atmosphere by the north polar cap. After sublimating from the cap, HDO is transported along with water toward the equator. Figure 2 shows indeed the contours of the summer high concentrations of HDO dipping into the northern tropics as the season proceeds. During the fall and winter of both hemispheres, the HDO concentrations of the high-latitude regions dramatically drop. In the polar nights, the low atmospheric temperatures imply a negligible vapor pressure and thus a water holding capacity significantly reduced.

[19] For a detailed comprehension of the water cycle, which basis can be applied to understand qualitatively that of HDO, the reader is referred to previous studies [Houben et al., 1997; Richardson and Wilson, 2002; Montmessin et al., 2004]. Here, we will mostly focus on the relative differences between the $\mathrm{H}_{2} \mathrm{O}$ and the HDO cycle.

\subsection{Seasonal Variation of the $\mathrm{D} / \mathrm{H}$ Ratio}

[20] In order to emphasize the different behaviors of $\mathrm{HDO}$ and $\mathrm{H}_{2} \mathrm{O}$, we present in Figure 2 the seasonal and geographic changes of the $\mathrm{D} / \mathrm{H}$ ratio in the vapor phase. For commodity, the $\mathrm{D} / \mathrm{H}$ ratio is expressed with respect to the Standard Mean Ocean Water value (hereafter SMOW). As mentioned previously, we have forced the north polar cap reservoir with a deuterated content corresponding to a value of 5.6 wrt. SMOW. Accordingly, one would expect to obtain the same value in the polar cap atmosphere during summer when water vapor sublimates. As shown by Figure 2, this is not the case. Our predictions indicate an atmospheric $\mathrm{D} / \mathrm{H}$ being significantly lower than what we prescribed in the cap (5 versus 5.6 wrt. SMOW). This is an interesting result since it implies that the north permanent cap is not able to supply the same isotopic concentration to the atmosphere as the one trapped in surface ice (at least in our model). We shall see later that water cold-trapping on the south residual cap is partly responsible for this result.

[21] As detailed by Montmessin et al. [2004], our water cycle model predicts that an important concentration of lowlying clouds (typically in the first kilometer) covers the polar cap in summer. Such clouds are favored by a high relative humidity in the lowest atmospheric layers (where water vapor is injected) and by diurnal temperature variations which, in spite of their weak amplitude, force the atmosphere to frequently exceed saturation. In turn, the formation of these clouds leads to an active fractionation effect which tends to concentrate HDO in the clouds, leaving the vapor phase depleted from a significant fraction of its deuterium content (see Figure 3). One could assimilate this phenomenon as an isotopic filter reducing the extraction of HDO from the cap during the sublimation season.

[22] If this isotopic filter turned out to be as efficient in reality as it is in our model, then one would have to reconsider the actual deuterium concentration in the north polar cap and therefore the mean deuterium concentration in Martian water.

[23] Simultaneous measurements of both $\mathrm{H}_{2} \mathrm{O}$ and HDO are scarce and affected by two major shortcomings. First, most of the observations encompass a large fraction of the Martian disk. A second problem is that since HDO has not yet been observed from space, the observations are affected by a poor atmospheric transmission in the vicinity of the Martian $\mathrm{H}_{2} \mathrm{O}$ and $\mathrm{HDO}$ lines, due to terrestrial water. Hence $\mathrm{D} / \mathrm{H}$ measurements suffer from large uncertainties, as summarized in Figure 4. Within the error bars, D/H measurements are essentially constant throughout a Martian year. As shown by Figure 4, our GCM simulations predict only slight seasonal variations $(\sim 2 \%)$ of the planetary-averaged $\mathrm{D} / \mathrm{H}$ ratio despite the global condensation/sublimation cycle which forces humidity to change seasonally by a factor of 2. The large humidity fluctuations reflect the occurrence of

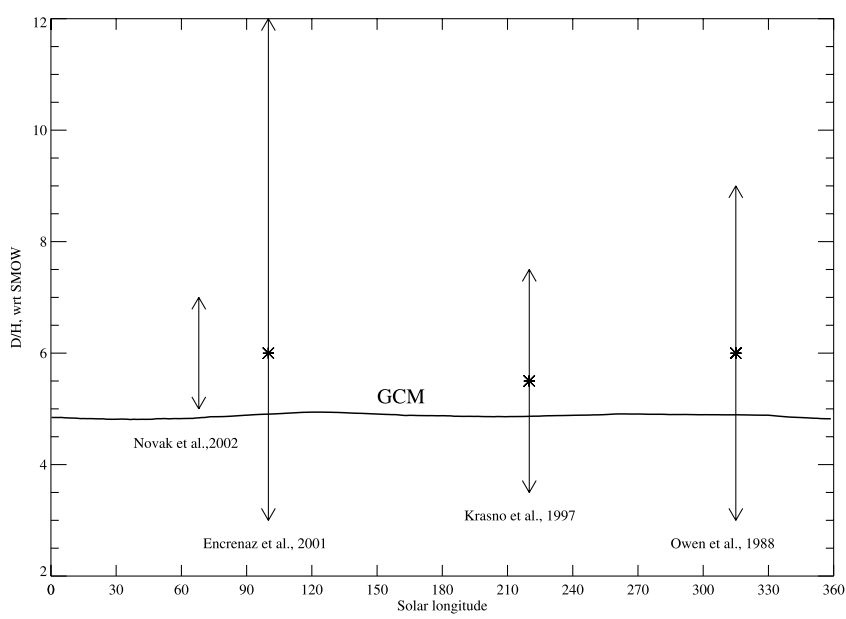

Figure 4. A comparison between various observations of the atmospheric $\mathrm{D} / \mathrm{H}$ ratio in the Martian atmosphere [Owen et al., 1988; Krasnopolsky et al., 1997; Encrenaz et al., 2001; Novak et al., 2002] and the seasonal evolution of the planetary-averaged $\mathrm{D} / \mathrm{H}$ predicted by the GCM. 


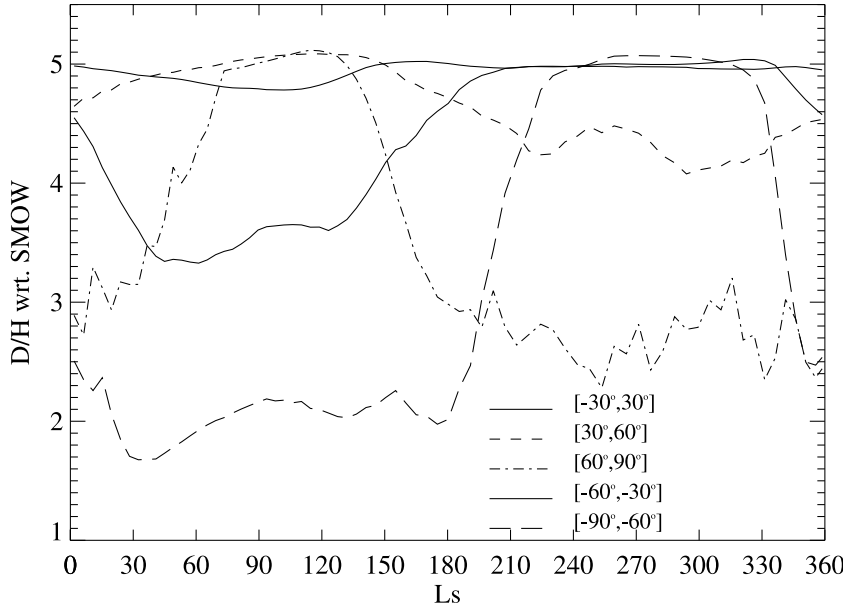

Figure 5. Seasonal evolution of $\mathrm{D} / \mathrm{H}$ in different latitudinal bands as obtained by the model.
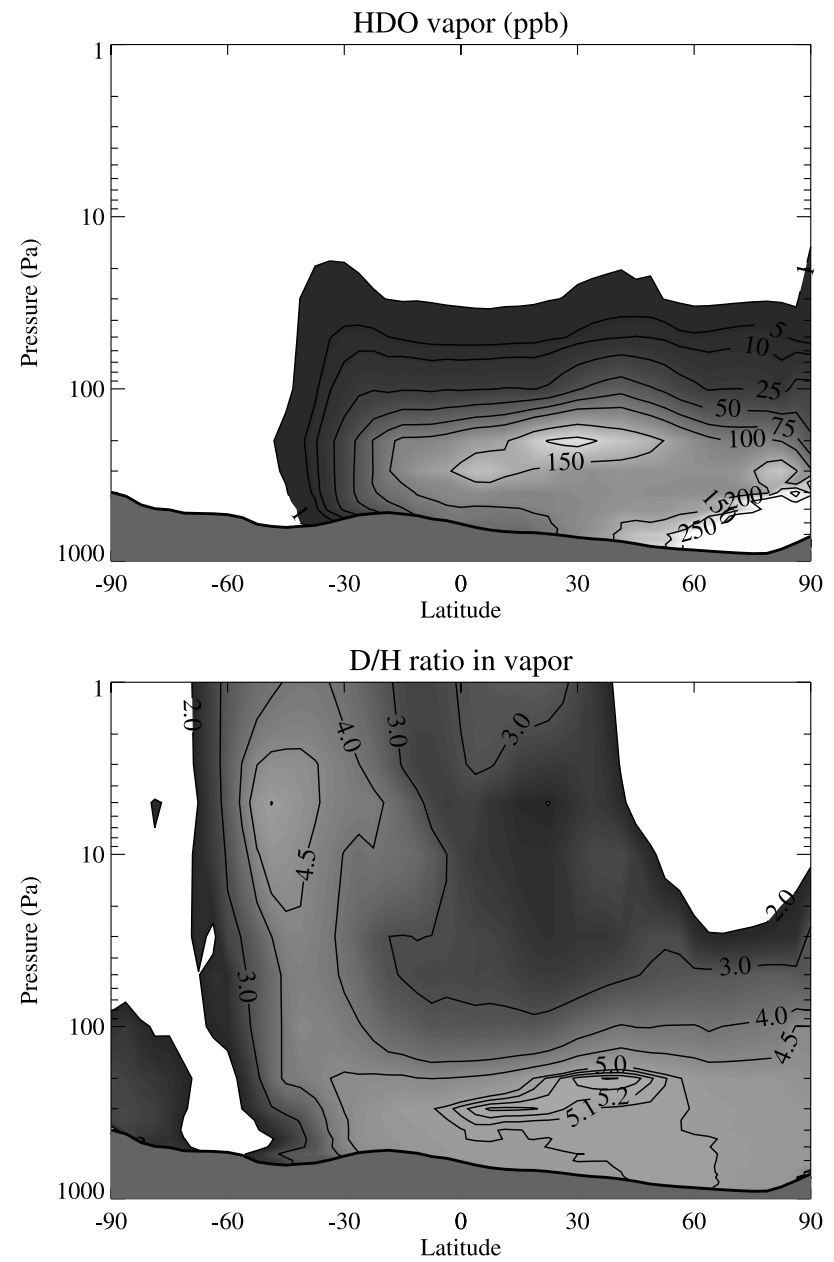

massive and local condensation episodes in the polar regions. As a result, HDO fractionation concentrates its effect locally and on a negligible remaining amount of water vapor so that a reduction in $\mathrm{D} / \mathrm{H}$ can not be sensed on a global scale.

\subsubsection{Aphelion Season Near the Equator}

[24] As displayed in Figure 5, the deuterium concentration in the latitudinal band $\left[30^{\circ} \mathrm{S}, 30^{\circ} \mathrm{N}\right]$ remains steady throughout the Martian year, with a mild deflation in the $\mathrm{D} / \mathrm{H}$ curve around northern summer solstice. This phenomenon is driven by the current orbital configuration of Mars, since the near aphelion period corresponds to a minimum of insolation in the equatorial region. This reduced insolation comes along with a lower dust loading, both leading to a significant decrease of atmospheric temperatures. This period has already been well documented [Clancy et al., 1996; James et al., 1996; Wolff et al., 1999; Liu et al., 2003], and the cold aphelion climate is now recognized to be at the origin of the Equatorial Cloud Belt (hereafter ECB) that has been observed in the $\left[10^{\circ} \mathrm{S}, 30^{\circ} \mathrm{N}\right]$ latitudinal band. The offset of the cloud belt toward the northern hemisphere is due to a convergence of water vapor in the lower atmosphere of the northern tropics where the overturning circulation possesses its rising branch (see Figure 6 for a
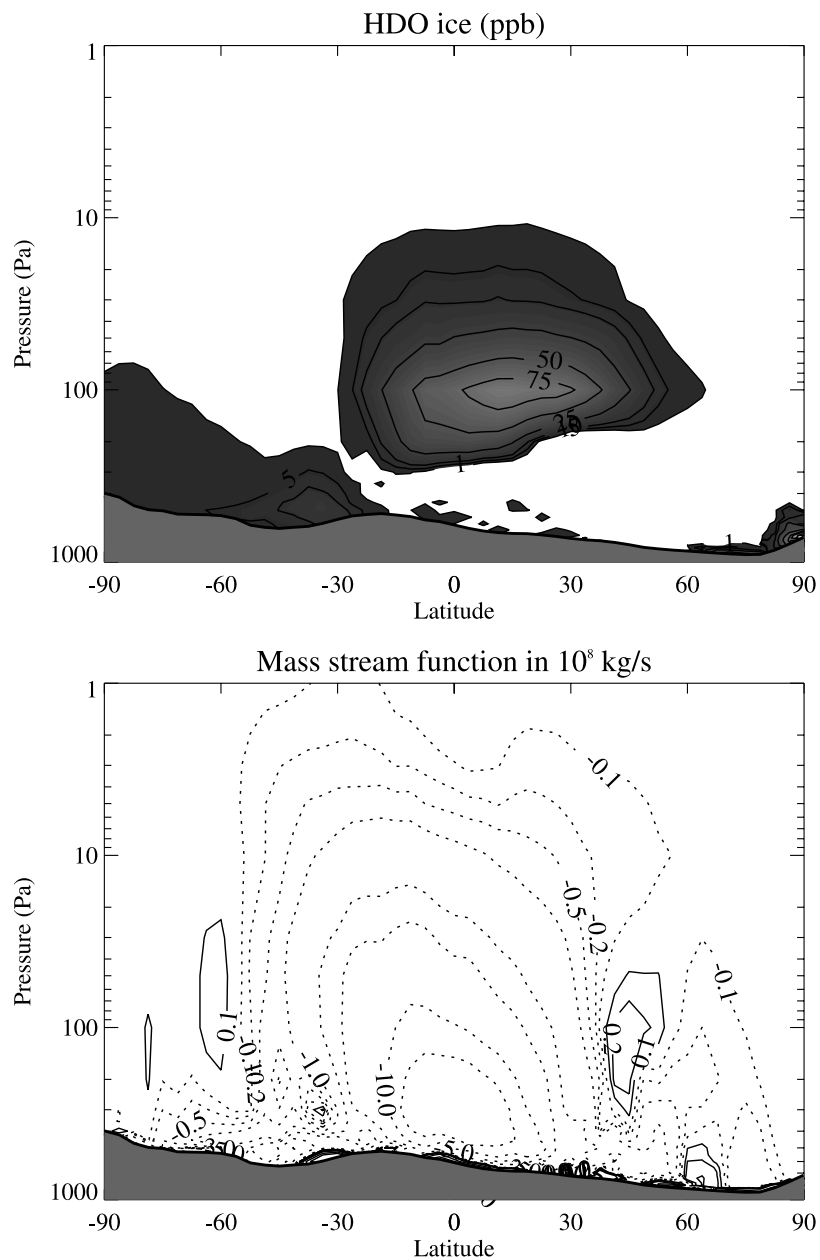

Figure 6. Meridional cross sections of HDO vapor, HDO ice, and D/H during northern summer $\left(\mathrm{L}_{s}=\right.$ $\left.90^{\circ}\right)$. As is the case for water, HDO condenses at low altitude in the equatorial region. The mass-stream function is also plotted to indicate the major patterns of the circulation at that season (negative values indicate a counterclockwise orientation of transport). 

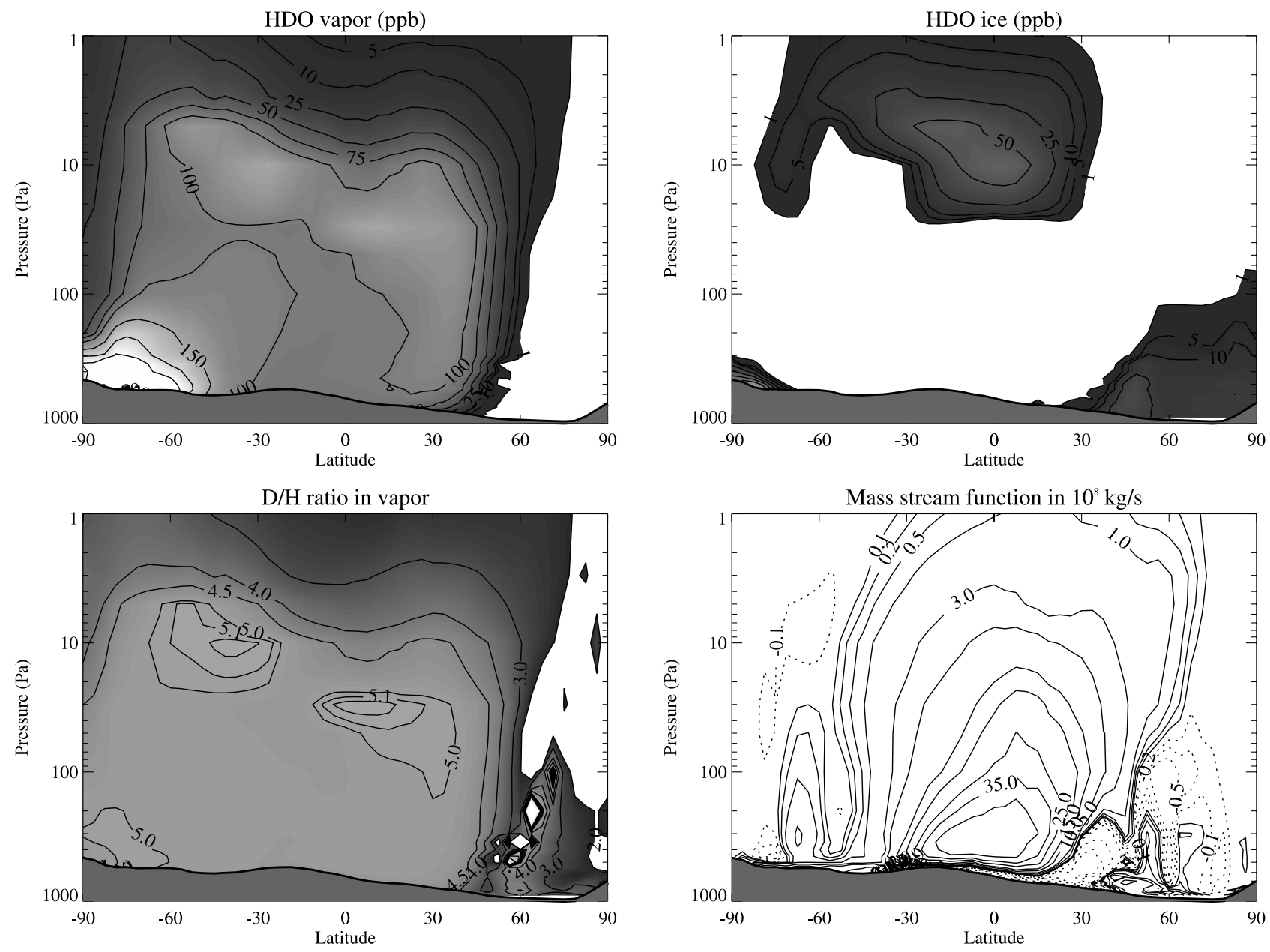

Figure 7. Same as Figure 6 but for southern summer $\left(\mathrm{L}_{s}=270^{\circ}\right)$.

plot of the mass-stream function). Here again, the formation of the ECB implies a depletion in HDO vapor in the equatorial region and therefore leads to the minimum in the $\mathrm{D} / \mathrm{H}$ curve of Figure 5 .

[25] Furthermore, the presence of the ECB in the upwelling zone of the Hadley cell has been shown by Richardson et al. [2002] and Montmessin et al. [2004] to significantly reduce the advection of water to the southern hemisphere by blocking the water flow under the hygropause of the northern tropics. In the case of HDO, this effect is even reinforced by the condensation-induced fractionation effect which tends to remove deuterium from the vapor phase and concentrate it in the clouds. The subsequent sedimentation and sublimation of these clouds creates a deuterium pump that transfers HDO molecules from above the hygropause to the atmosphere below it. This effect is illustrated by Figure 6, which shows a strong pooling of deuterium in the lower atmosphere of the northern tropics. Consequently, the vapor that is effectively carried to the southern hemisphere through the cloud belt gets significantly depleted in deuterium. This mechanism explains why Figure 2 exhibits a decrease in $\mathrm{D} / \mathrm{H}$ ratio in the $\left[30^{\circ} \mathrm{S}, 0^{\circ}\right]$ region as we approach northern summer solstice.

[26] The same mechanism works around southern summer solstice (Figure 7), but the hygropause occurs at such a high elevation that the bulk of the water vapor is essentially unaffected by the removal of HDO molecules into the clouds. For the same reason, the transfer of HDO from the south to the north via the Hadley cell is only affected at high altitudes (note that a similar pooling of deuterium occurs around $10 \mathrm{~Pa}$ in the $\left[60^{\circ} \mathrm{S}, 30^{\circ} \mathrm{S}\right]$ in Figure 7 ), allowing the northern hemisphere to be more efficiently supplied in HDO than the southern hemisphere at the opposite season.

[27] Additionally, Figure 6 indicates a local deuterium enrichment in the mid to high latitudes of the southern hemisphere at high altitude, where the $\mathrm{D} / \mathrm{H}$ ratio exceeds 4.5 near the pressure level $10 \mathrm{~Pa}(\sim 35-40 \mathrm{~km})$. At this altitude, however, the corresponding water vapor mixing ratio is almost negligible, but this local enrichment remains persistent down to lower heights near the polar vortex boundary of the southern hemisphere. By comparing this figure with the corresponding pattern of the mass stream function, we can see that this atmospheric portion of enriched deuterium is located within the descending branch of the Hadley cell. The straightforward explanation for this particular feature involves the contribution of the cloud belt. As shown by Montmessin et al. [2004], a substantial fraction of the clouds in the ECB are likely to be transported by the overturning circulation, participating, though less efficiently than vapor, to the supply of water to the southern hemisphere. When the clouds reach the downwelling region of the southern hemi- 
sphere, they progressively sublimate during their descending motion as air masses get adiabatically warmed. While releasing their deuterium content, arguably higher than that of the surrounding vapor, they create a zone of relatively larger $\mathrm{D} / \mathrm{H}$ ratios. This southward transport of icy $\mathrm{HDO}$ partially compensates for the reduced transport of HDO vapor that is blocked by the cloud belt in the northern tropics. Again, some evidence of a similar phenomenon occurs during the opposite season $\left(\mathrm{L}_{s} \sim 270^{\circ}\right)$, explaining the high deuterium ratios of the atmosphere in the $\left[0,30^{\circ} \mathrm{N}\right]$ latitudinal band around $50 \mathrm{~Pa}$.

\subsubsection{HDO in the Polar Regions}

[28] Figure 2 indicates a drastic decrease of the $\mathrm{D} / \mathrm{H}$ ratio in the polar regions during fall and winter. As stated earlier, the low atmospheric temperatures reigning in these regions imply very low water vapor pressure. The subsequent formation of the Polar Hood clouds generates a strong fractionation of HDO. This process depletes the vapor of the polar regions from a large fraction of its deuterium content, concentrating it in the icy particles. As these clouds tend to precipitate, HDO accumulate on the surface of the seasonal caps. Houben et al. [1997] and Richardson and Wilson [2002] have detailed a mechanism allowing most of the water incorporated in the cold seasonal $\mathrm{CO}_{2}$ cap to be carried back to the pole during the cap recession. This "quasi-solid" return of the water to the polar region also affects $\mathrm{HDO}$, though in a more efficient way, as the water ice in the seasonal cap is slightly enriched in deuterium. The release of this deuterium-rich water in the atmosphere during the second half of the spring season explains the progressive increase of $\mathrm{D} / \mathrm{H}$ ratios in the vicinity of the pole even before the permanent cap has started subliming (Figure 2).

[29] Consistent with Figure 2, Figure 5 shows that, closer to the pole, the amplitude of the seasonal $\mathrm{D} / \mathrm{H}$ variations increases. For instance, the $\left[30^{\circ} \mathrm{N}, 60^{\circ} \mathrm{N}\right]$ region sees its $\mathrm{D} / \mathrm{H}$ vary seasonally from 4 to 5 , whereas the changes in the $\left[60^{\circ} \mathrm{N}, 90^{\circ} \mathrm{N}\right]$ region fluctuate between 3 and 5 . The $\mathrm{D} / \mathrm{H}$ latitudinal distribution at several seasons is displayed in Figure 8. As already shown by Figure 5, the linear decrease in deuterium concentration within the fall/winter highlatitudes is once again well captured. While minimum at high latitudes, the $\mathrm{D} / \mathrm{H}$ ratio exhibits a local maximum near the fall/winter poles. In the southern hemisphere, the amplitude of the peak increases until approximately $\mathrm{L}_{s}=$ $180^{\circ}$ and is centered around $80^{\circ} \mathrm{S}$. The phenomenon is less perceptible in the northern hemisphere but appears nonetheless at $\mathrm{L}_{s}=0^{\circ}$ and at $\mathrm{L}_{s}=225^{\circ}$. No clear interpretation can be made to explain the presence of these peaks, their seasonal behavior is somewhat chaotic and they seem to be controlled by seasonal shifts of both maxima and minima. 3.2.3. Comparison With Ground-Based Observations

[30] The reduction in atmospheric deuterium in fall/winter high-latitude regions has been recently documented by ground-based observations. Using the C-SHELL instruments, Mumma et al. [2003] observed HDO on several locations on the planet. They then used the TES inferred water column abundance to deduce $\mathrm{D} / \mathrm{H}$ ratios. The results indicate a significant decrease of the atmospheric $\mathrm{D} / \mathrm{H}$ ratio poleward of the midlatitude regions. Values as low as 2.5 wrt. SMOW have been recorded at $\mathrm{L}_{s}=155^{\circ}$ in significantly wet regions $\left(\mathrm{H}_{2} \mathrm{O}>16 \mathrm{pr} . \mu \mathrm{m}\right)$. This picture agrees well with our predictions. Still, model and observations disagree on the cut-off latitude poleward of which the $\mathrm{D} / \mathrm{H}$ ratio starts to decrease. In addition, a compilation of the C-SHELL data shows a negative correlation between water abundances and $\mathrm{D} / \mathrm{H}$ ratios, with $\mathrm{D} / \mathrm{H}$ values higher than 8 wrt. SMOW for water abundances lower than $10 \mathrm{pr}$. $\mu \mathrm{m}$. This is clearly at odds with our results, which are summarized in Figure 9. In general, we obtain a logarithmic increase of $\mathrm{D} / \mathrm{H}$ with water vapor abundances. This positive correlation does not hold at very low water contents $(<0.1$ pr. $\mu \mathrm{m})$, as our model indicates a strong scattering of $\mathrm{D} / \mathrm{H}$ values in this range of humidity. In the future, a more thorough comparison with additional observations (this time, both $\mathrm{HDO}$ and $\mathrm{H}_{2} \mathrm{O}$ will be simultaneously measured; M. J. Mumma, personal communication) will undoubtedly help us constrain our HDO representation. For instance, if the negative correlation was confirmed by later measurements, we could suspect the influence of the regolith, an issue that would therefore need to be addressed by our model.

\subsection{Deuterium Loss in the South Residual Cap}

[31] Due to the presence of the ever-cold $\mathrm{CO}_{2}$ south residual cap which imposes negligible water vapor pressure near its surface and therefore traps water molecules coming in its vicinity, the atmosphere loses irremediably a substantial amount of water each year. However, since the water cycle seeks by nature to achieve a steady state (at least in the models), this fraction lost from the annual water budget is compensated by an equivalent amount of water removed from the north permanent cap during the sublimation season [Richardson and Wilson, 2002]. This way, the global inventory of water (atmosphere+seasonal surface reservoir) does not change interannually. In the case of HDO, however, the picture is complicated by the influence of fractionation.

[32] According to the model, the global $\mathrm{D} / \mathrm{H}$ ratio in the atmosphere steadily decreases interannually during and after the spin-up stage of the simulation, whereas both water and heavy water were accruing until the water cycle reaches steady state. Evidence of this is given in Figure 10, which shows that $\mathrm{D} / \mathrm{H}$ is reduced by approximately $0.5 \%$ at the end of the year. This annual $\mathrm{D} / \mathrm{H}$ decrease is in fact an indication that HDO has not achieved a steady state yet as it gets relatively more trapped than water by the $\mathrm{CO}_{2}$ residual cap. In Figure 11, we report the seasonal behavior of $\mathrm{H}_{2} \mathrm{O}$ and HDO in and above the south residual cap. Approximately $0.5 \mathrm{~mm}$ of water is added each year to the surface of the cap. Most of the deposition occurs during the first half of the southern summer, while the deposited water exhibits a deuterium enrichment compared to the overlying water vapor. On the average, the water that is trapped in the cap is fractionated by a factor 1.15 compared to the global mean deuterium concentration in the atmosphere $(\mathrm{D} / \mathrm{H} \sim 5.5$ versus 4.85).

[33] The influence of this HDO trap at the south pole on the HDO cycle can be studied with a simplified model where the water cycle is represented by three different components. The first is the reservoir of water that is seasonally cycled in and out the polar cap. It encompasses water under various forms, whether vapor or clouds in the atmosphere as well as the seasonal water ice frost that forms 

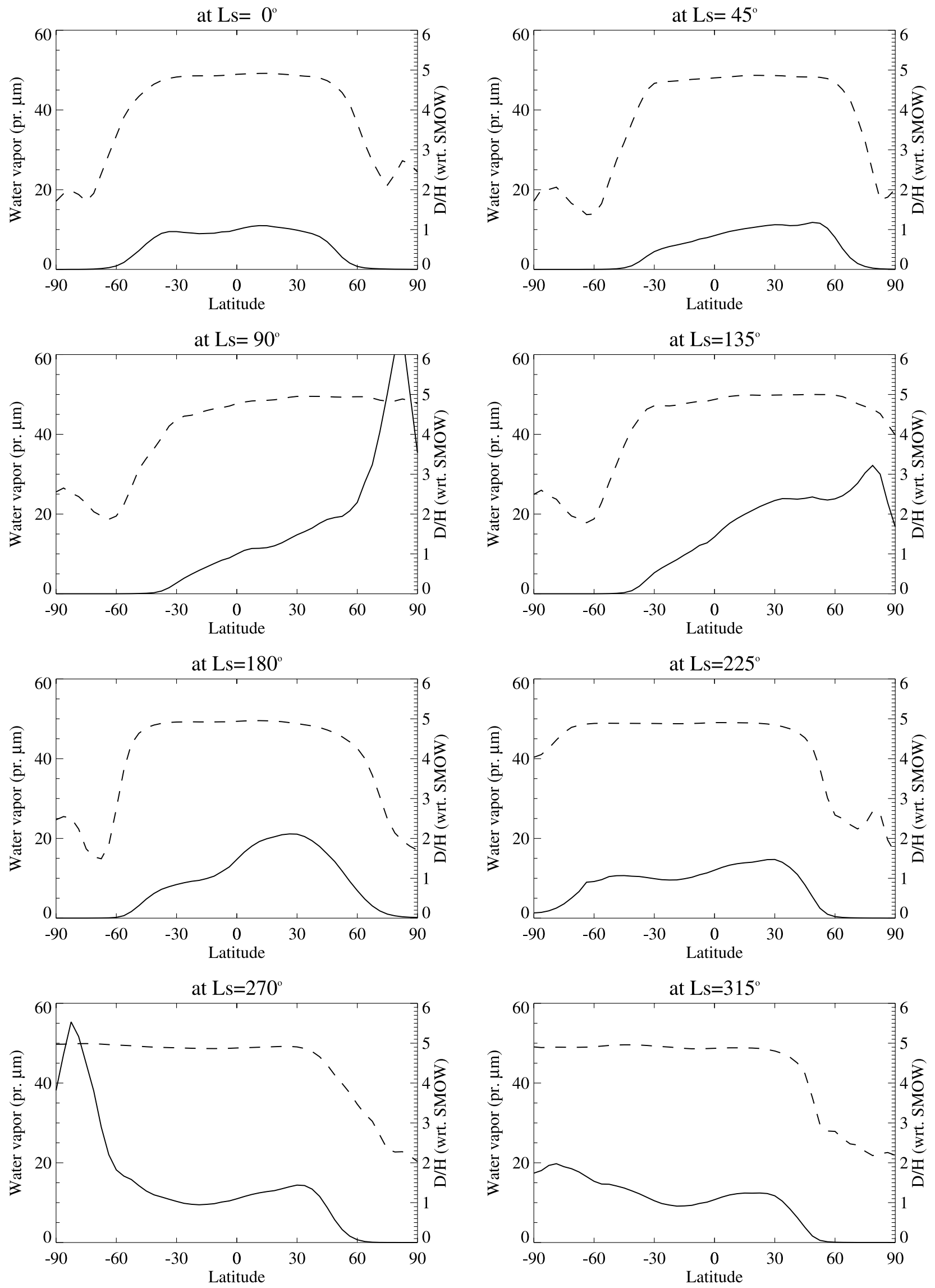

Figure 8. Latitudinal distributions of water vapor (solid curve) and D/H ratio (dashed curve) at several seasons. 


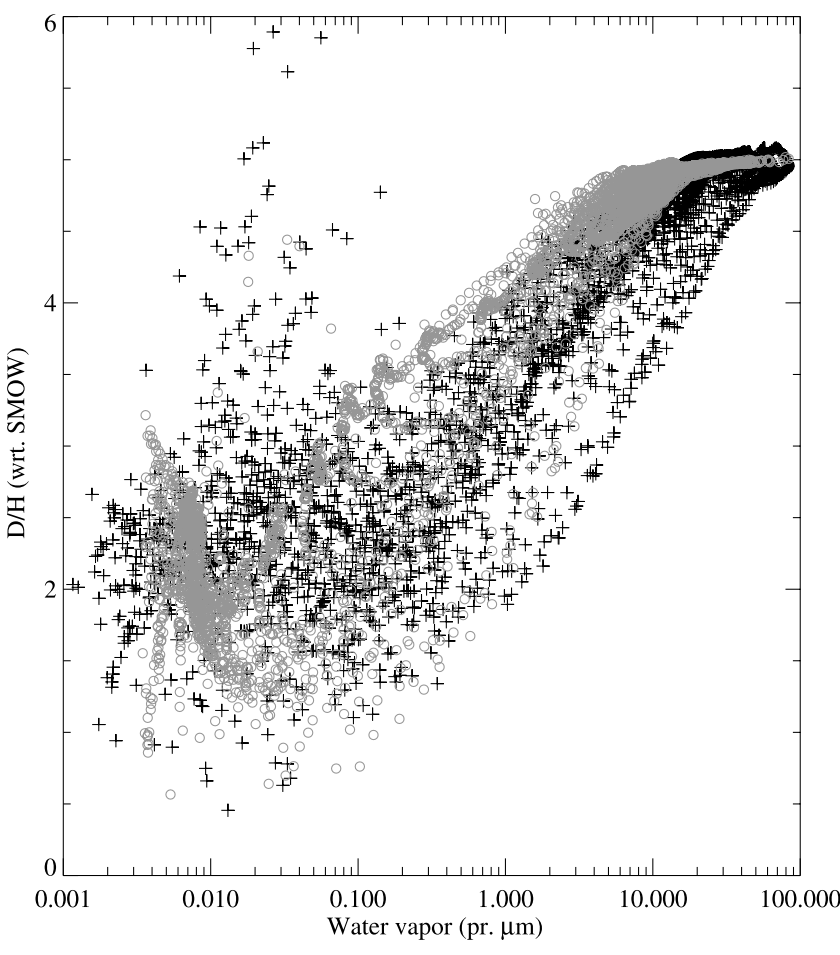

Figure 9. A compilation of zonally averaged water vapor abundances plotted against their corresponding $\mathrm{D} / \mathrm{H}$ ratios. The gray circles denote the values obtained in the southern hemisphere, whereas the dark crosses are for the northern hemisphere. Between 0.1 and $10 \mathrm{pr} . \mu \mathrm{m}, \mathrm{D} / \mathrm{H}$ logarithmically increases with humidity. At higher water vapor contents, the plots concentrate around a $\mathrm{D} / \mathrm{H}$ value of 5 . Note that the northern hemisphere exhibits a higher frequency of low $\mathrm{D} / \mathrm{H}$ in the $[0.1,10] \mathrm{pr} . \mu \mathrm{m}$ range.

and recedes in the polar regions during most part of the year. It represents an amount $Q_{a}$ of water. Each year during the spring and summer seasons when water vapor sublimates from the north permanent cap, an amount $\Delta Q$ is added to the first reservoir so as to compensate for the same $\Delta Q$ that is definitively lost by the system during southern summer on the south residual cap. The injected $\Delta Q$ that is drawn from the north cap represents the second component, whereas the lost $\Delta Q$ accounts for the third. As in this model and probably in reality, the north permanent cap gets eroded by this transfer of water from pole to pole, the water in the second component necessarily comes from the sublimation of an old ice that was deposited ages ago when the upper layers of the north residual cap were formed.

[34] In terms of deuterium, each of this component possesses a distinct isotopic ratio. For simplicity, we assume that the deuterium content $\delta_{o} \Delta Q$ of the second component reflects the isotopic concentration prevailing in the whole north permanent cap. In contrast, the deuterium content $\delta Q_{a}$ of the first component is supposed to fluctuate from year to year until the HDO cycle achieves its own steady state. And finally, the deuterium content $\delta_{l} \Delta Q$ of the last component includes a term of fractionation $\alpha$ which represents the influence of various processes that discriminate the deuterium concentration in the flux of water that is captured by the residual cap. These processes relate to any meteorolog- ical phenomenon that prevents the water that is locally trapped by the $\mathrm{CO}_{2}$ cap to have the same $\mathrm{D} / \mathrm{H}$ ratio as that of the other reservoirs; like for instance the fractionation of the water that is deposited onto the cap after condensation. We define $\alpha$ as $(\mathrm{D} / \mathrm{H})_{l}=\alpha(\mathrm{D} / \mathrm{H})_{a+o}$, where the index $l$ refers to the deuterium concentration of the third component, whereas the index $a+o$ refers to that of the sum of the first and the second components. We have summarized this model in the sketch of Figure 12.

[35] According to our description, the annual budget of HDO can be expressed as follows:

$$
\delta_{n} Q_{a}=\delta_{n-1} Q_{a}+\delta_{o} \Delta Q-\delta_{l} \Delta Q,
$$

where $\delta_{n} Q_{a}$ denotes the deuterium content of the first reservoir at the end of year $n$ after $\Delta Q$ has been added then removed to the system, whereas $\delta_{n-1} Q_{a}$ denotes the deuterium content of the first reservoir at the end of year $n$ -1 . Furthermore, $\delta_{l}$ can be related to $\alpha$ in the following way:

$$
\alpha=\frac{\delta_{l}}{\frac{\delta_{n-1} Q_{a}+\delta_{o} \Delta Q}{Q_{a}+\Delta Q}}
$$

such that

$$
\delta_{l}=\alpha \frac{\delta_{n-1} Q_{a}+\delta_{o} \Delta Q}{Q_{a}+\Delta Q}
$$

Let $x$ be the ratio $\Delta Q / Q_{a} ; \delta_{l}$ can thus be written as

$$
\delta_{l}=\alpha\left(\frac{x \delta_{o}}{1+x}+\frac{\delta_{n-1}}{1+x}\right)
$$

leading to

$$
\delta_{n}=\left(1-\frac{\alpha x}{1+x}\right)\left[x \delta_{o}+\delta_{n-1}\right]
$$

By expanding further, we can obtain a recursive relation between $\delta_{n}$ and the initial deuterium content $\delta_{o}$ of the atmosphere at year 0 (which we assume to be equal to that of the permanent cap):

$$
\frac{\delta_{n}}{\delta_{o}}=\left(1-\frac{\alpha x}{1+x}\right)^{n}+x \sum_{i=1}^{n}\left(1-\frac{\alpha x}{1+x}\right)^{i}
$$

As $n$ increases, the ratio $\left(\delta_{n} / \delta_{o}\right)$ gets closer to its equilibrium value. Indeed, for $n \rightarrow \infty$, we get

$$
\frac{\delta_{n}}{\delta_{o}}=\frac{1}{\alpha}[1+x(1-\alpha)]
$$

In other words, the difference in deuterium concentrations between the atmosphere and the ground source (i.e., the north permanent cap) will not change interannually once $n$ becomes sufficiently high.

[36] The lifetime of a water molecule before it gets eventually cold-trapped by the residual cap should vary as $1 / x$ (in years). Our model shows that approximately $2 \%$ of the global water inventory is seasonally captured by the south cap. The HDO cycle will therefore require more than 


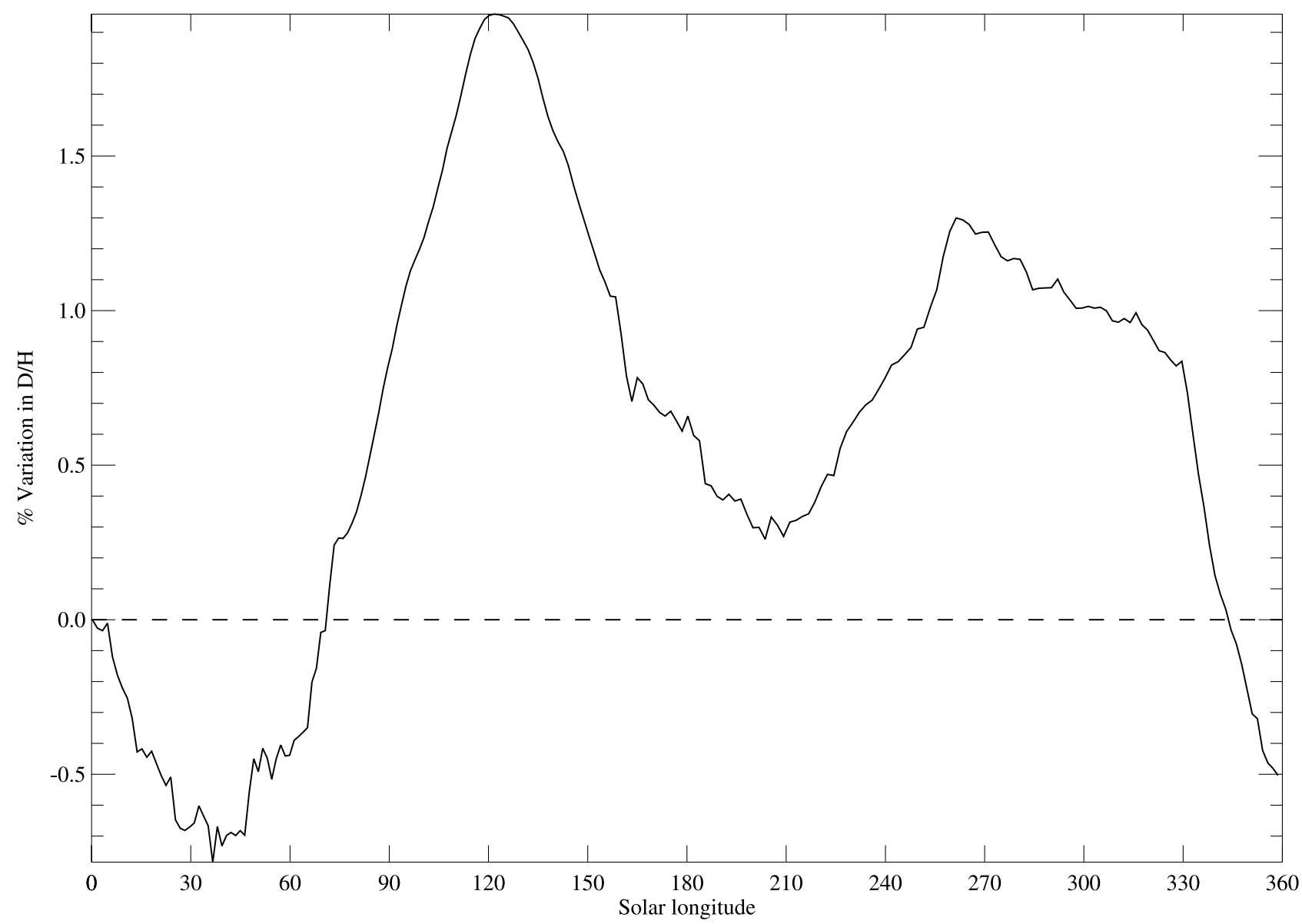

Figure 10. Seasonal evolution of the percentage of variation of the planetary-averaged $\mathrm{D} / \mathrm{H}$ ratio as deduced from the model. By the end of the year, $\mathrm{D} / \mathrm{H}$ has lost $0.5 \%$ of its initial value.

$n=1 / 0.02=50$ years to achieve its own steady state. A $2 \%$ annual loss of water is a rather conservative value since at the spatial resolution of our model, we underestimate the dimensions of the south cap by more than a factor of 2. Still, a 50 year timescale remains negligible in comparison of the Martian orbital timescale which exceeds several Martian millennia. But the age of the south residual cap remains uncertain. In theory, the decaying rate of the mesas in the "Swiss cheese" terrains of the south pole should permit the $\mathrm{CO}_{2}$ residual cap to survive several centuries [Byrne and Ingersoll, 2003]. This should leave ample time to the HDO cycle to achieve its steady state. However, Barker et al. [1970] measured unusually high amounts of water vapor in the southern summer hemisphere. This event was interpreted as the sublimation of a perennial water ice layer and thus could have been indicative of a partial or complete removal of the overlying $\mathrm{CO}_{2}$ residual cap. Within this context, it is still difficult to conclude that the present-day value of the atmospheric $\mathrm{D} / \mathrm{H}$ indeed reflects that of a transient HDO cycle or that of an equilibrated cycle.

[37] In Figure 13, we report the sensitivity of the ratio $\left(\delta_{n} /\right.$ $\delta_{o}$ ) to both $x$ and $\alpha$ as given by equation (3) (note that the figure illustrates well the spin-up timescale dependence on the fraction $x$ ). For the low $x$ values considered in our case, the influence of the south residual cap on the HDO cycle is mostly dependent on the value of $\alpha$ (as expressed by equation (4)). The cold-trapping effect appears clearly sig-
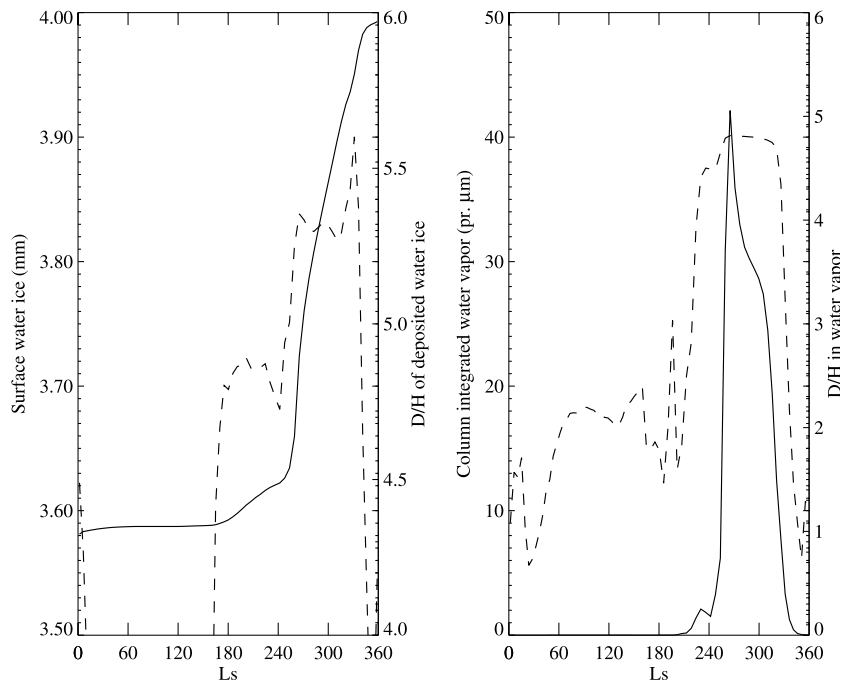

Figure 11. (left) Seasonal accumulation of water ice at the south pole (solid curve). The dashed line shows the instantaneous deuterium concentration in the flux of water depositing on the south residual cap. (right) Seasonal variation of the water vapor abundance above the south residual cap (solid curve). The dashed line is for the corresponding value of the $\mathrm{D} / \mathrm{H}$ ratio in the gas phase. 


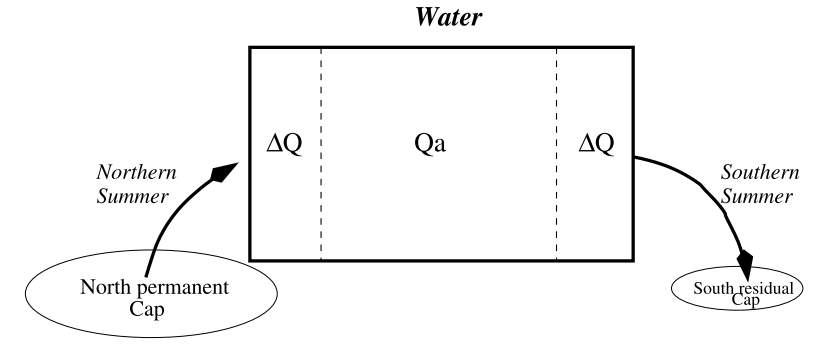

HDO

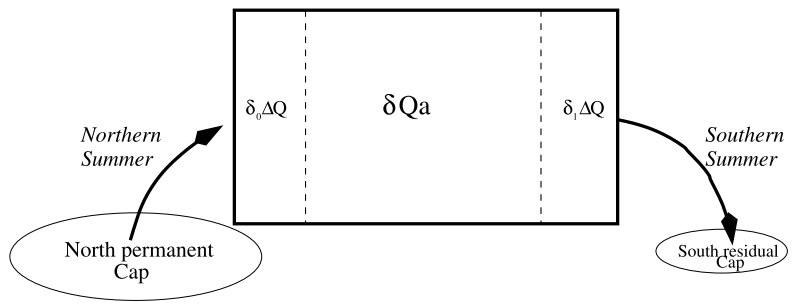

Figure 12. Chart describing the exchanges of water between the three major components of the water cycle: the north permanent cap, the seasonal reservoir (atmosphere and ground frost), and the south residual cap. In the upper part, we show the corresponding exchanges in HDO.

nificant since it can modulate the equilibrium value of the global atmospheric $\mathrm{D} / \mathrm{H}$ value by large factors. We have included $\alpha$ coefficients lower than unity. Whereas the model results contradict such a possibility $(\alpha \sim 1.15)$, it is interesting to note that on Earth, $\alpha<1$ is a configuration that usually dominates [Joussaume et al., 1984]. Indeed, due to the remote location of the water isotope source regions, a large amount of precipitation occurs in the air masses traveling to the pole. When the latter reach their destination, they already have been severely depleted from their deuterium content. Such a mechanism could also prevail on Mars if the bulk of the cold-trapped water in the south cap originated from air masses traveling through the polar night. According to the model, it is not the case; water comes in theory from the recession of the seasonal cap while some local fractionation makes the cold-trapped water richer in deuterium than the surrounding vapor (Figure 12).

[38] To our predicted $\alpha$ value of 1.15 corresponds a $\left(\delta_{n} /\right.$ $\delta_{o}$ ) ratio of roughly 0.85 . This implies in turn that the actual $\mathrm{D} / \mathrm{H}$ ratio in the north permanent cap should exceed 6.5 as opposed to the 5.6 observed in the atmosphere. While this difference does not fundamentally change our picture of Martian deuterium, it must be acknowledged that the influence of a putative deuterium sink may have modulated the evolution of the $\mathrm{D} / \mathrm{H}$ ratio through ages. Such a mechanism complicates our understanding of the fate of Martian deuterium, but this issue can be addressed in the more general context of orbitally induced climatic changes.

\section{Predicted Cycle of HDO: The Rapid Homogenization Case}

[39] In order to set a lower limit to the effect of HDO fractionation on a global scale, we now study the case where a thermodynamic equilibrium is assumed to take place between icy particles and the surrounding vapor. This configuration is envisioned as an alternative to the Rayleigh
Distillation case studied in the previous section since the isotopic relaxation time inside Martian-type cloud particles is theoretically not always slow enough for HDO molecular diffusion in ice to be neglected.

[40] A comparison between the two experiments $\mathrm{RD}$ and $\mathrm{RH}$ is given in Figure 14, where the two different HDO seasonal cycles have been ratioed. Globally averaged, the $\mathrm{RH}$ experiment produces HDO abundances $4 \%$ higher than in the $\mathrm{RD}$ case. In the polar regions, this percentage can be as high as $60 \%$ at some specific seasons and in particular during the formation and the recession stages of the $\mathrm{CO}_{2}$ seasonal caps. This pattern prevails in both hemispheres. The largest differences between the two models exactly correspond to the locations and to the seasons where the Polar Hood cloud formation is intensified [Montmessin et al., 2004]. During the formation of the seasonal caps, significant water vapor abundances still remain from previous spring/summer sublimation, whereas atmospheric temperatures start to decrease. In addition, the circulation organizes itself so as to produce an enhanced poleward transport of humidity from the wetter, equatorward regions. As the water holding capacity of the high-latitude atmosphere progressively shrinks, water ice clouds start to form. Airborne condensation will in turn deplete the surrounding vapor from its deuterated content. The HDO vapor depletion being sensitive to the type of fractionation chosen, $\mathrm{RH}$ and RD experiments produce significantly different results.

[41] During the recession of the caps, cloud formation is enhanced by a brutal increase in wave activity near the border of the $\mathrm{CO}_{2}$ caps. As shown by Montmessin et al. [2004], stationary and transient disturbances combine their actions to create an equatorward movement of cold air masses carrying thick clouds, whereas an opposite poleward motion of warm air transports the humidity recently released by the cap. The latter phenomenon is able to supply large amounts of condensible material to the Polar Hood clouds and thus reinforces their formation. Here again, this mech-
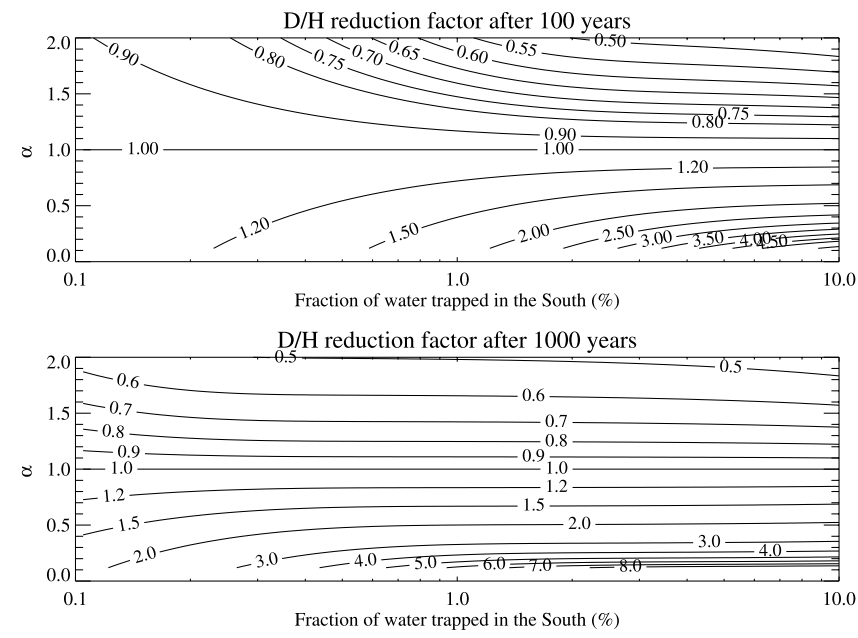

Figure 13. Ratios of the equilibrium value of $\mathrm{D} / \mathrm{H}$ in the Martian atmosphere onto that prevailing in the source region of the north residual cap. The ratio is plotted as a function of $x$, the fraction of the seasonal water reservoir that is lost each year in the south residual cap, and $\alpha$, the coefficient of deuterium enrichment in the water coldtrapped by the south residual cap. 


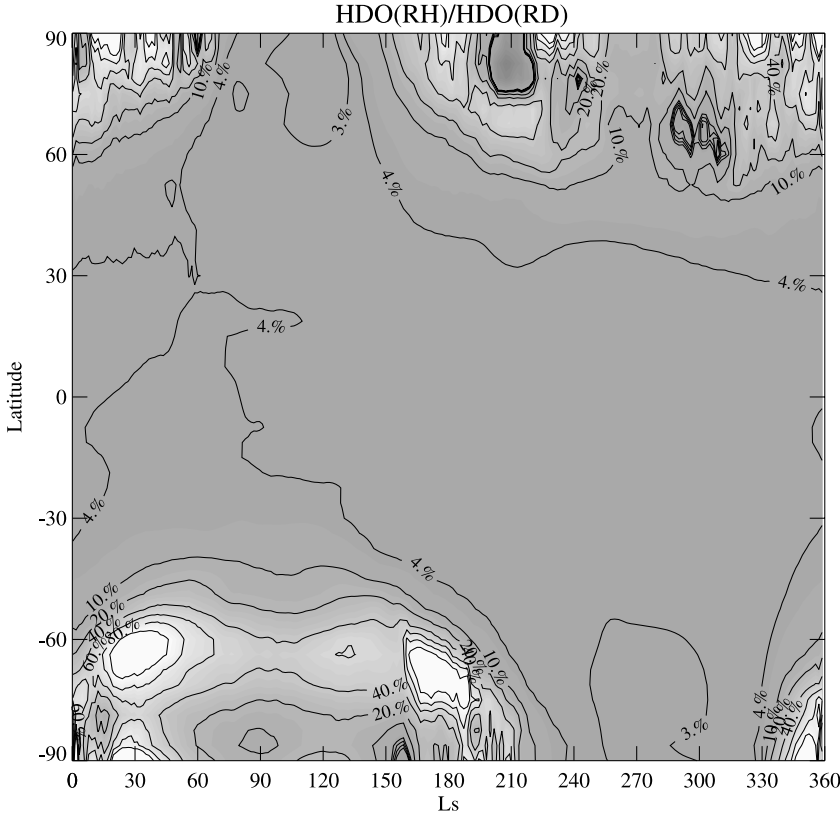

Figure 14. Seasonal and geographical variations of the HDO abundances predicted by the Rapid Homogenization model $(\mathrm{RH})$ onto those predicted by the Rayleigh Distillation model (RD).

anism results in a severe depletion of HDO, the magnitude of which being controlled by our different assumptions on fractionation.

[42] Nonetheless, the RD and the RH yields a qualitatively similar behavior of HDO. The nature of HDO cycle remains deeply controlled by the seasonal variation of insolation in the high-latitude regions. Despite the significantly different HDO fractionation treatment between the two experiments, we still obtain a picture of the HDO cycle where the equator to midlatitude regions exhibit a steady value of the $\mathrm{D} / \mathrm{H}$ ratio, whereas the fall/winter polar regions shows a significant decrease in $\mathrm{D} / \mathrm{H}$, with values as low as 3 (wrt. SMOW) in both cases.

[43] Another subject of interest concerns the deuterium loss in the south residual cap. In the previous section, we have shown that the $\mathrm{CO}_{2}$ permanent cap seasonally captures an amount of water enriched in deuterium. In the RH case, however, the deuterium enrichment is only $8 \%$ as opposed to the $15 \%$ previously obtained. As fractionation is treated in the same way in both experiments when water condenses directly on the ground, this result indicates that most of the water trapped by the residual cap originates from cloud precipitation. The south cap shows itself once again capable at differentiating HDO, but in a less pronounced manner that directly pertains to our restrained fractionation treatment. In this case, the resulting equilibrium $\mathrm{D} / \mathrm{H}$ value in the Martian atmosphere for present-day conditions should be $95 \%$ of that in the permanent cap.

\section{Vertical Distribution of HDO: Annual Mean Location of the Deuteropause}

[44] The vertical distribution of HDO is of considerable importance for the production of $\mathrm{D}$ atoms. As depicted in the introduction, the photodissociation of HDO molecules in the lower atmosphere leads ultimately to the presence of $\mathrm{D}$ atoms at high altitude, where they can more easily escape from the Martian gravity.

[45] Due to the fractionation effect at condensation, the air masses that move up through the hygropause are theoretically depleted from their deuterated species. Like the Earth, Mars should also possess a "deuteropause"; i.e., a level above which the $\mathrm{HDO} / \mathrm{H}_{2} \mathrm{O}$ ratio abruptly decreases. This was already predicted by Fouchet and Lellouch [2000] and by Bertaux and Montmessin [2001], and is now supported by the present study for some specific seasons (see Figures 6 and 7).

[46] In addition, Cheng et al. [1999] have shown that UV absorption by the $\mathrm{CO}_{2}$ molecules screens a portion of the solar spectrum where the HDO molecule cross section is larger than that of $\mathrm{H}_{2} \mathrm{O}$ molecules. At higher UV wavelengths, however, where the $\mathrm{CO}_{2}$ is essentially nonabsorbing, the opposite stands. Spectrally integrated, the photolysis differentiation between $\mathrm{HDO}$ and $\mathrm{H}_{2} \mathrm{O}$ favors the photodissociation of $\mathrm{H}_{2} \mathrm{O}$ molecules.

[47] Hence, should photolysis occur in the HDO-poor air masses located above the hygropause, the production of deuterium atoms will be dramatically reduced (a factor of 9 has been invoked by Bertaux and Montmessin [2001]), and so will be the propagation of D atoms to the upper atmosphere.

[48] This combination of fractionations provides an efficient means for the Martian atmosphere to retain its deuterium in comparison of its hydrogen. But as stated above, for this mechanism to work optimally, the hygropause (to which corresponds a deuteropause) needs to be located
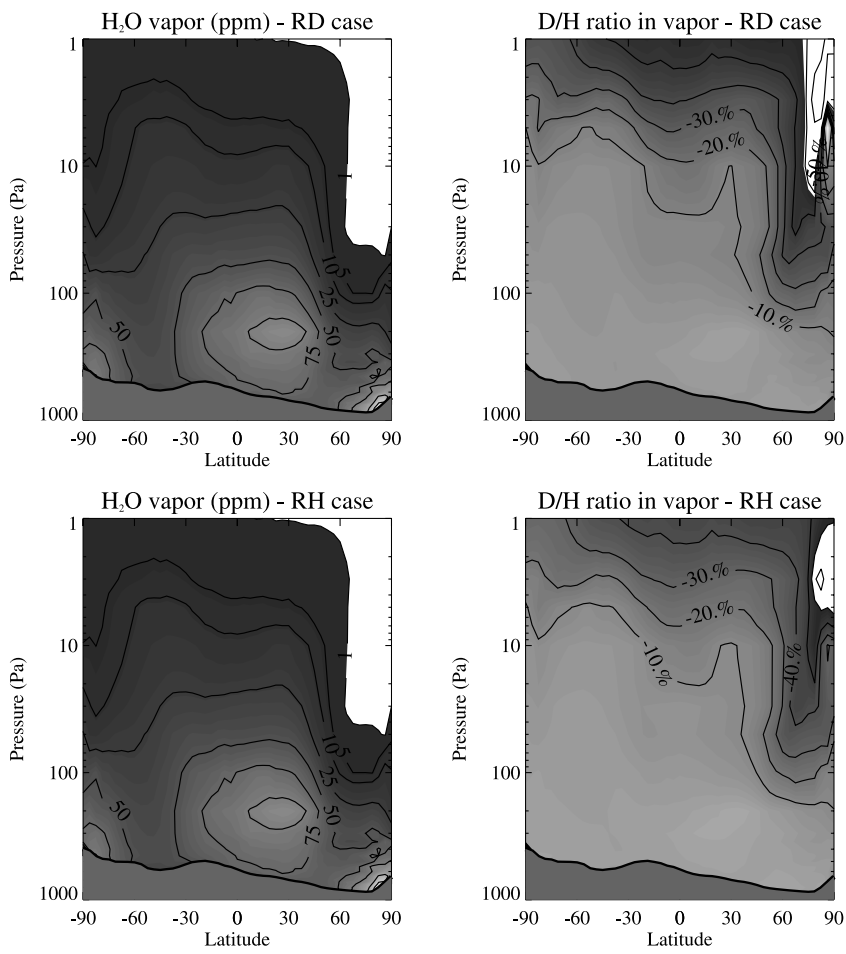

Figure 15. Meridional cross sections of the annually averaged water vapor concentration in the atmosphere and their corresponding $\mathrm{D} / \mathrm{H}$. D/H contours show the percentage of local departure from the global mean value. 

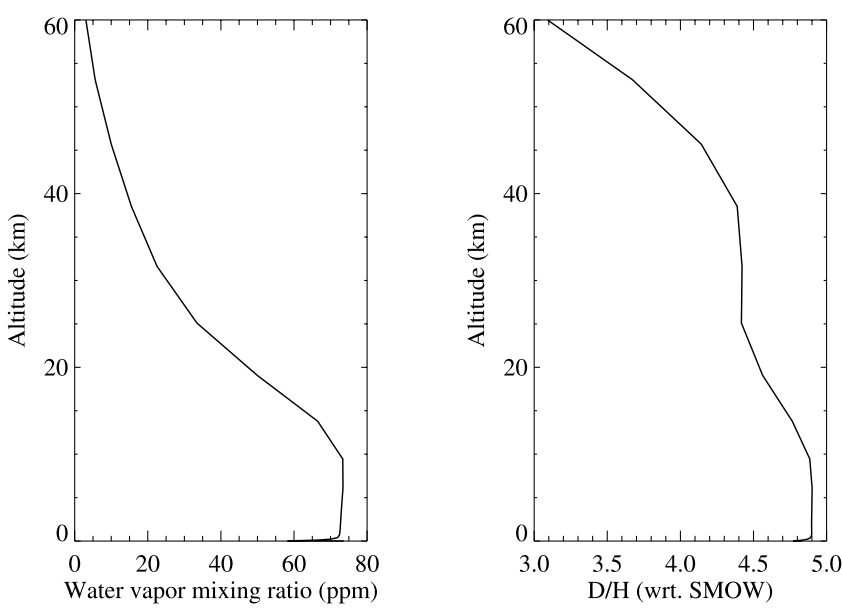

Figure 16. Globally and annually averaged vertical distributions of water vapor (on the left) and of the $\mathrm{D} / \mathrm{H}$ ratio (on the right).

below the photolysis bulk region $(\sim 25 \mathrm{~km}$ [Cheng et al., 1999]).

[49] In the absence of a photochemical modeling of the D species, we can not assess the validity of this mechanism on a global scale. However, our model predictions can be at least used to determine the average height of the deuteropause and compare it to that of the photolysis region.

[50] In Figure 15, we show the meridional cross sections of the annually averaged $\mathrm{H}_{2} \mathrm{O}$ concentrations and $\mathrm{D} / \mathrm{H}$ ratios in the atmosphere. The two different fractionation cases yield similar HDO distributions. A distinct asymmetry differentiates the northern hemisphere from the south. As shown by Figure 6 and Figure 7, the elevation of the deuteropause during the dustier southern summer in the south hemisphere is significantly higher than at the opposite season in the opposite hemisphere. This seasonal shift of the deuteropause is well rendered by the $\mathrm{D} / \mathrm{H}$ cross sections of Figure 15: it can be seen that the $\mathrm{D} / \mathrm{H}$ ratio starts to decrease only at heights higher than $40 \mathrm{~km}(\sim 10 \mathrm{~Pa})$ in the region covering the south pole to $30^{\circ} \mathrm{N}$. Northward of $30^{\circ} \mathrm{N}$, the deuteropause level falls abruptly to around $15 \mathrm{~km}(\sim 200 \mathrm{~Pa})$.

[51] The meridional average of Figure 15 is given in Figure 16. This plot represents the annual and global mean vertical distribution of water vapor and its corresponding $\mathrm{D} / \mathrm{H}$ value in the Martian atmosphere. The water vapor curve gradually decreases above $10-15 \mathrm{~km}$, whereas the D/H curve exhibits a two-step structure with a first decline around $15 \mathrm{~km}$ and a second one located near $40 \mathrm{~km}$. This two-step structure of $\mathrm{D} / \mathrm{H}$ reflects the influence of two distinct deuteropause behaviors in each hemisphere.

[52] The presence of HDO in significant quantities at altitudes greater than $30 \mathrm{~km}$ around perihelion effectively counterbalance the otherwise low concentrations at other seasons. Hence the question remains as to whether the fractionation coupling advocated by Bertaux and Montmessin [2001] is strong enough to explain the observed relative paucity of D atoms at high altitude [Krasnopolsky et al., 1998]. The results presented here are a major update of the vertical profiles of $\mathrm{HDO} / \mathrm{H}_{2} \mathrm{O}$ predicted by Fouchet and Lellouch [2000] and by Bertaux and Montmessin [2001]. Since those profiles were used by Krasnopolsky [2000] to constrain his photochemical model, it would be interesting to see what impact these new results may have on the predictions of the high-altitude $\mathrm{D}$ concentration.

\section{Conclusion}

[53] In this study, we have presented and discussed the first 3-D modeling of a water isotope in a planetary atmosphere other than Earths. Because HDO has a lower vapor pressure than $\mathrm{H}_{2} \mathrm{O}$, the condensed phase in equilibrium with vapor is enriched in the deuterated isotope. As a result, HDO is fractionated each time a water ice cloud is forming or when water is directly condensing onto the surface. It is thus of particular interest to see how this condensation-induced fractionation process does affect the HDO cycle on a global scale. The results of our work can be summarized as follows:

[54] - Despite the large seasonal variations of humidity, the global value of $\mathrm{D} / \mathrm{H}$ in the Martian atmosphere should remain steady, with only mild seasonal fluctuations in the 2 to $3 \%$ range.

[55] - This same ratio is, however, predicted to change by more than a factor of 2 in the mid- to high-latitude regions of both hemispheres due to the seasonal formation of the thick Polar Hood clouds.

[56] - Different types of fractionation models for condensation yield essentially the same qualitative behavior for the HDO cycle, with differences exceeding $10 \%$ only in regions of very low HDO abundances.

[57] - The presence of an Equatorial Cloud Belt around northern summer seems quite able to restrain the southward flow of HDO vapor compared to water vapor, but this effect is, however, compensated by the transport of deuterium-rich cloud particles.

[58] - The presence of a deuterium sink like the residual $\mathrm{CO}_{2}$ cap in the south should buffer the atmospheric mean $\mathrm{D} / \mathrm{H}$ ratio at equilibrium to a value $8 \%$ to $15 \%$ lower than that prevailing in the north permanent cap.

[59] - The annual mean vertical distribution of HDO does not exhibit a sharp decline above the hygropause like that previously predicted by 1-D models.

[60] This type of model opens the door to a wide range of experiments and improvements. First, the absence of a regolith adsorption scheme might be prejudicial for future comparisons with observations. Not only will condensation fractionate HDO when water ice is forming in the regolith pores, but the process of adsorption itself might account for an additional source of fractionation, as do all processes sensitive to molecular weight. Still, it is difficult to anticipate which role could play the regolith in the seasonal development of the HDO cycle as its role remains not understood for the current water cycle. On the other hand, a regolith scheme could help us reconcile with C-SHELL observations which exhibit a negative correlation between $\mathrm{D} / \mathrm{H}$ ratios and water vapor contents, whereas our model yields the opposite. On much longer timescales (thousand to million of years), Kass and Yung [1999] demonstrated the need for an additional reservoir of water (and heavy water) to buffer the global $\mathrm{D} / \mathrm{H}$ ratio to its observed value and considered the regolith as one of the possible candidates. In addition, large quantities of subsurface ice have been discovered in the high-latitude regions [Boynton et al., 
2002]. These features are now understood as resulting from a transition period in obliquity when water could have moved from the tropics and accumulated poleward of $60^{\circ}$ [Levrard et al., 2004]. Isotopic exchanges with a subsurface reservoir should thus have been a major component of the HDO history. In that light, a consistent representation of the regolith is highly desirable to investigate the evolution of the $\mathrm{D} / \mathrm{H}$ ratio over time.

[61] We have also neglected the effect of kinetic fractionation in our computations. Differences in molecular diffusivities between water and its isotope change the relative behavior of HDO during condensation if this process is in nonequilibrium. More precisely, the higher molecular mass of HDO makes it comparatively slower at diffusing from the gas phase to the surface of icy particles. This effect is mostly important in significantly supersaturated environments [Jouzel and Merlivat, 1984]. In this case, the lower HDO diffusivity balances the effect of its lower vapor pressure so as to reduce the relative enrichment in deuterium of the condensed phase. We think, however, that for kinetic fractionation to be consistently included in a HDO modeling, a more detailed representation of microphysics is needed. In particular, the interactions between dust and icy particles (like nucleation or scavenging) are in theory the major factor controlling the local degree of saturation. As in our model, these microphysical processes are not represented (instead dust is a prescribed quantity), our predictions of local supersaturation states are not consistent enough to be used for kinetic fractionation.

[62] In a near future, we plan to couple the photochemical model developed by Lefèvre et al. [2004] to our HDO model in order to simulate the production of deuterium atoms in the Martian atmosphere. The coupling of condensation and photolysis fractionation processes has been advocated to explain the low population of $\mathrm{D}$ atoms in the upper atmosphere [Bertaux and Montmessin, 2001]. However, our results suggest that this coupling might not be as efficient as 1-D models predict it. The high seasonal variability of the hygropause leads to the presence of large HDO concentrations at heights supposedly relevant to photodissociation. Nonetheless, this statement will have to be confirmed by a comprehensive deuterium cycle model where HDO photochemistry is explicitly represented.

[63] Also, it will be worth investigating the sensitivity of the HDO cycle to the orbital variations of Mars. It can be envisioned that different orbital configurations may lead to different equilibrium values of the atmospheric D/H. Furthermore, given the importance of the concentration of deuterium in the north permanent cap, we shall stress out the necessity of understanding the formation of this water ice reservoir and its implication in terms of deuterium. Among other questions, we may ask ourselves if the observed atmospheric $\mathrm{D} / \mathrm{H}$ ratio can be taken as representative of the whole deuterium concentration in the cap. Indeed, the formation of the north permanent cap might have been comparable to a distillation process. It is thus possible that HDO may have been segregated in the first layers deposited, with the cap gradually losing in HDO concentration as its thickness increased. Potentially, a much larger atmospheric concentration of deuterium would thus be observed if one were to sublime the entire north permanent cap. These questions have direct impacts on our assumptions on the total inventory of Martian deuterium, and through it on the amount of water escaped to space.

[64] On Earth, the variations with depth of $\mathrm{HDO} / \mathrm{H}_{2} \mathrm{O}$ inside the extracted water ice cores of the polar regions are used as a means to reconstruct the various climatic changes of the past. If in the future, a mission undertakes the drilling of ice cores in the Martian polar regions, similar information on the Martian climate may be deduced. On the other hand, we have shown in this study, that different processes control the transport and the deposition of HDO in the polar regions. Within this context, applying the same empirical law that relates Earth climate to the HDO concentrations at the poles may not be appropriate for Mars. As our model may be employed to simulate any given climatic scenario, we shall be able to establish a similar correlation but this time applicable to Mars. Such a model may thus be of a considerable interest to help interpreting future in-situ measurements of HDO.

[65] Acknowledgments. The authors thank Eric Chassefière for initiating discussions on the role of the south polar cap in the HDO cycle. This study was conducted while F.M. was holding a National Research Council award to do research at NASA/ARC.

\section{References}

Baker, V. R., M. H. Carr, V. C. Gulick, C. R. Williams, and M. S. Marley (1992), Channels and valley networks, in Mars, edited by H. H. Kieffer et al., pp. 493-522, Univ. of Ariz. Press, Tucson.

Barker, E. S., R. A. Schorn, A. Woszczyk, R. G. Tull, and S. J. Little (1970), Mars: Detection of atmospheric water vapor during the southern hemisphere spring and summer season, Science, 170, 1308-1310.

Bertaux, J., and F. Montmessin (2001), Isotopic fractionation through water vapor condensation: The Deuteropause, a cold trap for deuterium in the atmosphere of Mars, J. Geophys. Res., 106(E12), 32,879-32,884.

Boynton, W. V., et al. (2002), Distribution of hydrogen in the near surface of Mars: Evidence for subsurface ice deposits, Science, 297, 81-85.

Byrne, S., and A. P. Ingersoll (2003), A sublimation model for Martian south polar ice features, Science, 299, 1051-1053.

Cheng, B., E. Chew, C. Liu, M. Bahou, Y. Lee, Y. L. Yung, and M. F. Gerstell (1999), Photo-induced fractionation of water isotopomers in the Martian atmosphere, Geophys. Res. Lett., 26, 3657-3660.

Clancy, R. T., A. W. Grossman, M. J. Wolff, P. B. James, D. J. Rudy, Y. N. Billawala, B. J. Sandor, S. W. Lee, and D. O. Muhleman (1996), Water vapor saturation at low latitudes around aphelion: A key to Mars climate?, Icarus, 122, 36-62.

Dansgaard, W. (1964), Stable isotopes in precipitation, Tellus, 16, 436468 .

Encrenaz, T., E. Lellouch, G. Paubert, and S. Gulkis (2001), The water vapor vertical distribution on Mars from millimeter transitions of HDO and $\mathrm{H}_{2}^{18} \mathrm{O}$, Planet. Space Sci., 49, 731-741.

Forget, F., F. Hourdin, R. Fournier, C. Hourdin, O. Talagrand, M. Collins, S. R. Lewis, P. L. Read, and J. Huot (1999), Improved general circulation models of the Martian atmosphere from the surface to above $80 \mathrm{~km}$, J. Geophys. Res., 104, 24,155-24,176.

Fouchet, T., and E. Lellouch (2000), Vapor pressure isotope fractionation effects in planetary atmospheres: Application to deuterium, Icarus, 144, $114-123$.

Houben, H., R. M. Haberle, R. E. Young, and A. P. Zent (1997), Modeling the Martian seasonal water cycle, J. Geophys. Res., 102, 9069-9083.

Jakosky, B. M., and C. B. Farmer (1982), The seasonal and global behavior of water vapor in the Mars atmosphere: Complete global results of the Viking atmospheric water detector experiment, J. Geophys. Res., 87, 2999-3019.

James, P. B., J. F. Bell, R. T. Clancy, S. W. Lee, L. J. Martin, and M. J. Wolff (1996), Global imaging of Mars by Hubble Space Telescope during the 1995 opposition, J. Geophys. Res., 101, 18,883-18,890.

Joussaume, S., R. Sadourny, and J. Jouzel (1984), A general circulation model of water isotope cycles in the atmosphere, Nature, 311, 24-29.

Jouzel, J., and L. Merlivat (1984), Deuterium and oxygen 18 in precipitation: Modeling of the isotopic effects during snow formation, J. Geophys. Res., 89, 11,749-11,757.

Kass, D. M., and Y. L. Yung (1999), Water on Mars: Isotopic constraints on exchange between the atmosphere and surface, Geophys. Res. Lett., 26, $3653-3656$. 
Krasnopolsky, V. (2000), On the deuterium abundance on Mars and some related problems, Icarus, 148, 597-602.

Krasnopolsky, V. A., and P. D. Feldman (2001), Detection of molecular hydrogen in the atmosphere of Mars, Science, 294, 1914-1917.

Krasnopolsky, V. A., G. L. Bjoraker, M. J. Mumma, and D. E. Jennings (1997), High-resolution spectroscopy of Mars at 3.7 and $8 \mu \mathrm{m}$ : A sensitive search of $\mathrm{H}_{2} \mathrm{O}_{2}, \mathrm{H}_{2} \mathrm{CO}, \mathrm{HCl}$, and $\mathrm{CH}_{4}$, and detection of $\mathrm{HDO}$, J. Geophys. Res., 102(E3), 6525-6534.

Krasnopolsky, V. A., M. J. Mumma, and G. R. Gladstone (1998), Detection of atomic deuterium in the upper atmosphere of Mars, Science, 280, $1576-1580$.

Lefèvre, F., S. Lebonnois, F. Montmessin, and F. Forget (2004), Threedimensional modeling of ozone on Mars, J. Geophys. Res., 109, E07004, doi:10.1029/2004JE002268.

Leshin, L. A. (2000), Insights into Martian water reservoirs from analyses of Martian meteorite QUE94201, Geophys. Res. Lett., 27(14), 20172020

Levrard, B., F. Forget, F. Montmessin, and J. Laskar (2004), Recent ice-rich deposits formed at high latitudes on Mars by sublimation of unstable equatorial ice during low obliquity, Nature, 431, 1072-1075.

Liu, J., M. I. Richardson, and R. J. Wilson (2003), An assessment of the global, seasonal, and interannual spacecraft record of Martian climate in the thermal infrared, J. Geophys. Res., 108(E8), 5089, doi:10.1029/ 2002JE001921.

Mangold, N., Q. Quantin, V. Ansan, C. Delacourt, and P. Allemand (2004), Evidence for precipitation on Mars from dendritic valleys in the Valles Marineris area, Science, 305(5680), 78-81 doi:10.1126/ science. 1097549

Merlivat, L., and G. Nief (1967), Fractionnement isotopique lors des changements d'états solide-vapeur et liquide-vapeur de l'eau à des températures inférieures à $0^{c} \mathrm{C}$, Tellus, 19(1), 122-127.

Michelangeli, D. V., O. B. Toon, R. M. Haberle, and J. B. Pollack (1993), Numerical simulations of the formation and evolution of water ice clouds in the Martian atmosphere, Icarus, 100, 261-285.

Montmessin, F., F. Forget, P. Rannou, M. Cabane, and R. M. Haberle (2004), Origin and role of water ice clouds in the Martian water cycle as inferred from a general circulation model, J. Geophys. Res., 109 E10004, doi:10.1029/2004JE002284.

Mumma, M. J., R. E. Novak, M. A. Disanti, B. Bonev, N. dello Russo, and K. Magee-Sauer (2003), Seasonal mapping of $\mathrm{HDO}$ and $\mathrm{H}_{2} \mathrm{O}$ in the
Martian atmosphere, in Sixth International Conference on Mars, p. 3186, Lunar and Planet. Inst., Houston, Tex.

Novak, R. E., M. J. Mumma, M. A. DiSanti, N. D. Russo, and K. MageeSauer (2002), Mapping of ozone and water in the atmosphere of Mars near the 1997 aphelion, Icarus, 158, 14-23.

Owen, T., J. P. Maillard, C. de Bergh, and B. L. Lutz (1988), Deuterium on Mars-The abundance of HDO and the value of D/H, Science, 240, $1767-1770$.

Richardson, M. I., and R. J. Wilson (2002), Investigation of the nature and stability of the Martian seasonal water cycle with a general circulation model, J. Geophys. Res., 107(E5), 5031, doi:10.1029/2001JE001536.

Richardson, M. I., R. J. Wilson, and A. V. Rodin (2002), Water ice clouds in the Martian atmosphere: General circulation model experiments with a simple cloud scheme, J. Geophys. Res., 107(E9), 5064, doi:10.1029/ 2001JE001804.

Smith, M. D. (2002), The annual cycle of water vapor on Mars as observed by the Thermal Emission Spectrometer, J. Geophys. Res., 107(E11), 5115, doi:10.1029/2001JE001522.

Smith, M. D. (2004), Interannual variability in TES atmospheric observations of Mars during 1999-2003, Icarus, 167, 148-165.

Watson, L. L., I. D. Hutcheon, S. Epstein, and E. M. Stolper (1994), Water on Mars-Clues from deuterium/hydrogen and water contents of hydrous phases in SNC meteorites, Science, 265, 86-90.

Wolff, M. J., J. F. Bell, P. B. James, R. T. Clancy, and S. W. Lee (1999), Hubble Space Telescope observations of the Martian aphelion cloud belt prior to the Pathfinder mission: Seasonal and interannual variations, J. Geophys. Res., 104, 9027-9042.

Yung, Y. L., J. Wen, J. P. Pinto, K. K. Pierce, and M. Allen (1988), HDO in the Martian atmosphere-Implications for the abundance of crustal water, Icarus, 76, 146-159.

F. Forget, Laboratoire de Météorologie Dynamique, Institut Pierre Simon Laplace, 4 Place Jussieu, F-75252 Paris Cedex 05, Paris, France.

T. Fouchet, LESIA, Observatoire de Paris, 5 Place Jules Jansen, 92125 Meudon Cedex, Paris, France.

F. Montmessin, Service d'Aèronomie, CNRS/IPSL, Aile 45-46, 4ème Etage, Boite 102, 4 Place Jussieu, 75252 Paris Cedex 05, France. (montmes@aero.jussieu.fr) 